\title{
Stabilized Conforming Nodal Integration: Exactness and Variational Justification
}

\author{
K.Y.Sze ${ }^{1 *}$, J.S.Chen ${ }^{2}$, N.Sheng ${ }^{1}$, X.H.Liu ${ }^{1}$
}

\begin{abstract}
SUMMARY
In most Galerkin mesh-free methods, background integration cells partitioning the problem domain are required to evaluate the weak form. It is therefore worthwhile to consider these methods using the notions of domain decomposition with the integration cells being the subdomains. Presuming that the analytical solution is admissible in the trial solution, domain and boundary integration exactness, which depend on the orders of the employed trial solution and the required solution exactness, are identified for the strict satisfaction of traction reciprocity and natural boundary condition in the weak form. Unfortunately, trial solutions constructed by many mesh-free approximants contain non-polynomial terms which cannot be exactly integrated by Gaussian quadratures. Recently, stabilized conforming (SC) nodal integration for Galerkin mesh-free methods was proposed and illustrated to be linearly exact. This paper will discuss how linear exactness is ensured and how spurious oscillation encountered by direct nodal integration is suppressed in SC nodal integration from a domain decomposition point of view. Moreover, it will be shown that SC nodal integration can be formulated by the Hellinger-Reissner Principle and thus justified in the classical variational sense. Applications of the method to straight beam, plate and curved beam problems are presented.
\end{abstract}

Keywords: mesh-free, nodal integration, integration, Galerkin, hybrid-stress, stabilized conforming

1 Department of Mechanical Engineering, The University of Hong Kong, Pokfulam Road, Hong Kong SAR, P.R.CHINA

2 Department of Civil \& Environmental Engineering, University of California, Los Angeles, 5713G Boelter Hall, CA90095-1593, USA

* Correspondence author. Email: kysze@hku.hk 


\section{INTRODUCTION}

Mesh-free methods have been very actively pursued by the research community of computational mechanics in the last decade. Broadly speaking, mesh-free methods can be categorized into Galerkin mesh-free methods [1-5], Petrov-Galerkin mesh-free methods [6] and collocation meshfree methods [7-9]. As integration of the weak form is necessary in Galerkin mesh-free methods, background integration cells are required. It is worthwhile to consider these methods using the notion of domain decomposition with the integration cells viewed as the subdomains partitioning the domain. Presuming that the analytical solution is admissible in the trial solution, the mechanism that the Galerkin method reproduces the analytical solution is studied. Though the weak form does not need to be integrated exactly, certain domain and boundary integration exactness requirements that depend on the orders of the trial solution and the desired order of solution exactness are identified for the strict fulfillment of the traction reciprocity and the natural boundary condition in the weak form. Unfortunately, commonly-used mesh-free approximants including those constructed by moving least-squares and reproducing kernel particle methods [2,3] contain non-polynomial components which cannot be exactly integrated by Gaussian quadratures.

To reduce the effort on defining the integration cells, large rectangular and triangular cells and high order quadratures are normally employed. The practice becomes cumbersome if the domain boundary is not straight. An alternative way to evaluate the weak form is the nodal integration whose core idea is to use nodes as the integration sampling points. By using the nodes as the representative points in Dirichlet tessellation, which is an "equal-distant rule" (see Figure 1), the nodal subdomains are the Voronoi cells [10]. Though the system matrix is non-singular, numerical solutions obtained by this "direct nodal integration" may be plagued by spurious oscillation which also occurs in the finite difference method. This phenomenon is often explained by the observation that the nodal gradient is independent of the nodal parameters at the same node. Beissel and Belystchko stabilized the oscillation by penalizing the weak form with the square of the equilibrium residue [11]. The same stabilized nodal integration was later employed by Bonet and Kulasegaram [12] who adopted the smoothed particle hydrodynamics approximation. Nevertheless, the equilibrium residual involves the second derivatives which are rather expensive to compute.

Instead of using the nodal gradient to compute the weak form of the nodal subdomains, Chen and his coworkers recently employed the smoothed nodal gradient [13-15] which, via the divergence theorem, is evaluated by boundary integration. In this manner, the trial solution needs to be evaluated on the boundary of the nodal subdomains only. The whole formulation does not involve any derivatives of the trial solution. Furthermore, the nodal integration can yield linear exactness and the oscillation occurring in the direct nodal integration is not observed. For the above 
reasons, the former integration scheme is termed the stabilized conforming (SC) nodal integration.

The paper is organized as follows. Section 2 presents two sufficient conditions for securing the solution exactness in Galerkin domain decomposition method. Section 3 briefly introduces the direct and stabilized nodal integrations. Section 4 presents how linear exactness is secured in the SC nodal integration. Interestingly, the conditions identified in Section 2 are not required in the SC nodal integration and only a much less demanding boundary integration consistency is necessary. Section 5 points out that the SC integration method can be formulated by the Hellinger-Reissner Principle and thus justified in the classical variational sense. Section 6 presents the numerical results for some one-dimensional, straight beam, plate and curved beam problems.

\section{DOMAIN DECOMPOSITION AND INTEGRATION EXACTNESS}

The terms "meshless", "mesh-free" and "element-free" generally refer to the ability of constructing approximations or interpolations without referring to meshes. They stand a sharp contrast to finite element method whose trial solution quality is very sensitive to the mesh regularity. Nevertheless, Galerkin mesh-free methods often require a background mesh or integration subdomains for evaluating the domain integrals appearing in the weak form. In a broad sense, domain decomposition is involved. It is therefore worthwhile to examine Galerkin mesh-free methods by the concepts of domain decomposition. The two-dimensional elasticity problem is selected to be the subject of discussion.

The two-dimensional domain under consideration and its entire boundary are denoted by $\Omega$ and $\partial \Omega$, respectively. Moreover, $\partial \mathrm{W}=\mathrm{G}_{u} \cup \mathrm{G}_{t}$ and $\mathrm{G}_{u} \cap \mathrm{G}_{t}=$ null where $\Gamma_{u}$ and $\Gamma_{t}$ are prescribed with the essential boundary condition (e.b.c.) and the natural boundary condition (n.b.c.). $\Omega$ is partitioned into subdomains $\Omega^{e}$, s such that $\cup \mathrm{W}=\mathrm{W}$ and $\mathrm{W} \cap \mathrm{W}=$ null for $a \neq b$. With displacement $\mathbf{u}=\left\{u_{1}, u_{2}\right\}^{T}$ taken to be the only field variable whereas displacement compatibility and e.b.c. taken to be prerequisites, the strong form of the domain decomposition problem can be summarized as:

- equilibrium condition: $\boldsymbol{L}^{T} \mathbf{C}(\boldsymbol{L} \mathbf{u})+\overline{\mathbf{b}}=\mathbf{0} \quad$ in $\Omega^{e,} \mathrm{~s}$

- n.b.c.: $\mathbf{n C}(\boldsymbol{L u})=\overline{\mathbf{t}} \quad$ on all $\mathrm{G}_{t}^{e}$ 's $\left(\mathrm{G}_{t}^{e}=\mathrm{G}_{t} \cap \partial \mathbf{W}\right)$

- traction reciprocity: $(\mathbf{n C}(\boldsymbol{L u}))^{m+}+(\mathbf{n C}(\boldsymbol{L u}))^{m-}=\mathbf{0}$ on all $\mathbf{G}_{m}$ 's $\left(\mathbf{G}_{m}=\partial \mathbf{W}^{n+} \cap \partial \mathbf{W}^{n-}\right)$

where 


$$
\boldsymbol{L}=\left[\begin{array}{cc}
\partial / \partial x_{1} & 0 \\
0 & \partial / \partial x_{2} \\
\partial / \partial x_{2} & \partial / \partial x_{1}
\end{array}\right], \mathbf{n}=\left[\begin{array}{ccc}
n_{1} & 0 & n_{2} \\
0 & n_{2} & n_{1}
\end{array}\right] \text { and }\left\{\begin{array}{l}
n_{1} \\
n_{2}
\end{array}\right\} \text { is the boundary outward unit normal. }
$$

Moreover, $\mathbf{C}$ is the material elasticity matrix, $\overline{\mathbf{b}}$ is the prescribed body force and $\overline{\mathbf{t}}$ is the prescribed boundary traction on $\mathrm{G}_{t}$. Superscripts " $m+$ " and " $m$-" designate any pair of adjacent subdomains and $\Gamma_{m}$ denotes their common boundary segment as portrayed in Figure 1. Imposition of the e.b.c. can be stated as:

$$
\mathbf{u}=\overline{\mathbf{u}} \quad \text { on all } \mathrm{G}_{u}^{e} \text { 's }\left(\mathrm{G}_{u}^{e}=\mathrm{G}_{u} \cap \partial \mathrm{W}\right)
$$

in mesh-free methods is not as straight forward as in the finite element method but can always be done. Displacement compatibility requires

$$
\mathbf{u}^{m+}=\mathbf{u}^{m-} \quad \text { on all } \mathrm{G}_{m} \text { 's. }
$$

The trial solution can always be expressed as:

$$
\mathbf{u}=\mathbf{N q}
$$

where $\mathbf{N}$ is the shape function matrix and $\mathbf{q}$ is the vector comprising all nodal parameters or d.o.f.s. The following potential energy functional can be adopted:

$$
\mathbf{P}=\mathbf{q}^{T} \sum_{e}\left[\left\langle\frac{1}{2}(\boldsymbol{L} \mathbf{N})^{T} \mathbf{C}(\boldsymbol{L} \mathbf{N}) \mathbf{q}-\mathbf{N}^{T} \overline{\mathbf{b}}\right\rangle_{\mathrm{W}}-\left\langle\mathbf{N}^{T} \overline{\mathbf{t}}\right\rangle_{\mathrm{G}_{t}^{e}}\right]
$$

where $\langle\circ\rangle_{\mathrm{W}}$ and $\langle\circ\rangle_{\mathrm{G}}$ denote the numerically integrated counterparts of the exact integrals $\int_{\mathrm{W}} \circ \mathrm{dW}$ and $\int_{G} \circ d G$, respectively. Variation of $\Pi$ is:

$$
\mathrm{dP}=\mathrm{d} \mathbf{q}^{T} \sum_{e}\left[\left\langle(\boldsymbol{L} \mathbf{N})^{T} \mathbf{C}(\boldsymbol{L} \mathbf{N}) \mathbf{q}-\mathbf{N}^{T} \overline{\mathbf{b}}\right\rangle_{\mathrm{W}}-\left\langle\mathbf{N}^{T} \overline{\mathbf{t}}\right\rangle_{\mathrm{G}_{t}^{e}}\right]
$$

The subdomain boundary $\partial \mathrm{W}$ can be partitioned into $\mathrm{G}_{u}^{e}, \mathrm{G}_{t}^{e}$ and the portion shared with the adjacent subdomains. Recalling that e.b.c. is a prerequisite (i.e. $\delta \mathbf{u}=\mathbf{0}$ on $\mathrm{G}_{u}^{e}$ ), the divergence theorem can be written as:

$$
\left\langle(\boldsymbol{L} \mathbf{N})^{T} \mathbf{C}(\boldsymbol{L} \mathbf{N})\right\rangle_{\mathrm{W}}=-\int_{\mathrm{W}} \mathbf{N}^{T}\left[\boldsymbol{L}^{T} \mathbf{C}(\boldsymbol{L} \mathbf{N})\right] d \mathbf{W}+\int_{\partial \mathrm{W} / \mathrm{G}_{u}^{e}} \mathbf{N}^{T} \mathbf{n C}(\boldsymbol{L N}) d \mathbf{G}+\mathbf{E}^{e}
$$


in which $\mathbf{E}^{e}$ is the error induced by the numerical integration and $\int$ denotes the exact integration as previously specified. By virtue of (9), (8) can be written as:

$$
\begin{aligned}
\mathrm{dP}_{P}= & \left.\mathrm{d} \mathbf{q}^{T} \sum_{e}\left(-\int_{W} \mathbf{N}^{T}\left[\boldsymbol{L}^{T} \mathbf{C}(\boldsymbol{L} \mathbf{N})\right] d \mathbf{W q}-\left\langle\mathbf{N}^{T} \overline{\mathbf{b}}\right\rangle_{W}+\int_{\mathrm{G}_{t}^{e}} \mathbf{N}^{T} \mathbf{n C}(\boldsymbol{L} \mathbf{N}) d \mathbf{G q}-\left\langle\mathbf{N}^{T} \overline{\mathbf{t}}\right]\right\rangle_{\mathrm{G}_{t}^{e}}+\mathbf{E}^{e} \mathbf{q}\right) \\
& +\mathrm{d} \mathbf{q}^{T} \sum_{m}\left(\int_{\mathrm{G}_{m}}\left[\mathbf{N}^{T} \mathbf{n C}(\boldsymbol{L} \mathbf{N})\right]^{m+} d \mathbf{G}+\int_{\mathrm{G}_{m}}\left[\mathbf{N}^{T} \mathbf{n C}(\boldsymbol{L} \mathbf{N})\right]^{m-} d \mathrm{G}\right) \mathbf{q}
\end{aligned}
$$

If the analytical solution is admissible in the trial solution, one would expect that the former will be predicted by Galerkin methods. However, this may not be the case when numerical integration is employed. The most trivial way ensuring that the analytical solution can be reproduced is to evaluate all terms in (7) exactly. Unfortunately, such quadratures, which are based on polynomial integrand, may not exist when the trial solution is based on mesh-free approximants. On the other hand, the integrands leading to the element stiffness matrices of distorted finite elements cannot be exactly integrated numerically due to the presence of the reciprocal of the Jacobian determinant as well. However, these elements pass the patch test and are linearly exact. In this light, exact integration of the weak form is not a necessity.

To examine the minimum quadrature for securing the k-th order exactness, $\mathbf{q}, \overline{\mathbf{b}}$ and $\overline{\mathbf{t}}$ in $\mathrm{dP}_{P}$ are set to respectively $\tilde{\mathbf{q}}, \tilde{\mathbf{b}}$ and $\mathbf{n C}(\boldsymbol{L N}) \tilde{\mathbf{q}}$ such that $\mathbf{u}=\mathbf{N} \tilde{\mathbf{q}}$ and $\overline{\mathbf{b}}=\tilde{\mathbf{b}}$ satisfy (1) whereas the order of $\mathbf{u}$ is $\mathrm{k}$. Thus,

$$
\left.\mathrm{dP}_{P}\right|_{\mathbf{q}=\tilde{\mathbf{q}}, \overline{\mathbf{b}}=\tilde{\mathbf{b}}, \overline{\mathbf{t}}=\mathbf{n} \mathbf{s}}=\mathrm{d} \mathbf{q}^{T} \sum_{e}\left(-\int_{W} \mathbf{N}^{T}\left(\boldsymbol{L}^{T} \tilde{\mathbf{s}}\right) d \mathbf{W}-\left\langle\mathbf{N}^{T} \tilde{\mathbf{b}}\right\rangle_{\mathrm{W}}+\int_{\mathrm{G}_{t}^{e}} \mathbf{N}^{T} \mathbf{n} \tilde{\mathbf{s}} d \mathbf{G}-\left\langle\mathbf{N}^{T} \mathbf{n} \tilde{\mathbf{s}}\right\rangle_{\mathrm{G}_{t}^{e}}+\mathbf{E}^{e} \tilde{\mathbf{q}}\right)
$$

in which $\tilde{\mathbf{s}}$ denotes $\mathbf{C}(\boldsymbol{L N}) \tilde{\mathbf{q}}$. As displacement compatibility is a prerequisite and "W" denotes exact integration, the last summation term in (10) vanishes identically and disappears in (11). Provided that the system matrix is non-singular, the sufficient conditions for $\mathbf{q}=\tilde{\mathbf{q}}, \overline{\mathbf{b}}=\tilde{\mathbf{b}}$ and $\overline{\mathbf{t}}=\mathbf{n} \tilde{\mathbf{s}}$ as the unique solution of the weak form are:

- domain integration exactness (DIE): $\left\langle(\boldsymbol{L} \mathbf{N})^{T} \tilde{\mathbf{s}}\right\rangle_{\mathrm{W}}=\int_{\mathrm{W}}(\boldsymbol{L} \mathbf{N})^{T} \tilde{\mathbf{s}} d \mathbf{W}$

- boundary integration exactness (BIE): $\left\langle\mathbf{N}^{T} \mathbf{n} \tilde{\mathbf{s}}\right\rangle_{\mathrm{G}_{t}^{e}}=\int_{\mathrm{G}_{t}^{e}} \mathbf{N}^{T} \mathbf{n} \tilde{\mathbf{s}} d \mathbf{G}$

It should be remarked that DIE implies $\mathbf{E}^{e} \tilde{\mathbf{q}}$ in (11) equal to zero. Since the order of $\mathbf{N}^{T} \tilde{\mathbf{b}}$ is always smaller than or equal to that of $(\boldsymbol{L N})^{T} \tilde{\mathbf{s}}$, DIE also ensures that the prescribed body force term is evaluated exactly and thus the pairwise cancellation of the two domain integration terms in (11). On the other hand, BIE leads to the pairwise cancellation of the boundary terms in (11). 
Noticeably, a higher order trial displacement or, equivalently, its shape function matrix $\mathbf{N}$ would require higher order quadrature rules to secure DIE and BIE regardless of how fine the integration subdomains are. Finally, the minimal quadrature for DIE and that for exact integration of $(\boldsymbol{L N})^{T} \mathbf{C}(\boldsymbol{L N})$ are different. The following examples illustrate the importance of DIE and BIE.

Example 2.1: To illustrate the importance of DIE, the following one-dimensional zero and constant body force problems are considered:

$$
\begin{aligned}
& \text { problem 1: } \frac{d^{2} u}{d^{2} x}=0 \text { for } 0 \leq x \leq 6,\left.u\right|_{x=0}=0,\left.\mathrm{~s}\right|_{x=6}=1 \Rightarrow u=x \text { and } \mathrm{s}=1 \\
& \text { problem 2: } \frac{d^{2} u}{d^{2} x}+1=0 \text { for } 0 \leq x \leq 6,\left.u\right|_{x=0}=0,\left.\mathrm{~s}\right|_{x=6}=1 \Rightarrow u=-x^{2} / 2+7 x \text { and } \mathrm{s}=-x+7
\end{aligned}
$$

where $\sigma=d u / d x$. The following trial solutions that comprise the exact solutions and satisfy the e.b.c. are employed:

$$
u_{1}^{h}=c_{1} x+c_{2} x^{2}, u_{2}^{h}=c_{1} x+c_{2} x^{2}+c_{3} x^{3} \text { and } u_{3}^{h}=c_{1} x+c_{2} x^{2}+c_{3} x^{3}+c_{4} x^{4}
$$

which require at least 2, 3 and 4 integration points to secure a non-singular system matrix, respectively. Given a quadrature rule, the above numbers of integration points are secured by partitioning the domain into equal-sized integration subdomains.

For problem 1, the orders of $(\boldsymbol{L N})^{T} \tilde{\mathbf{s}}$ 's for $u_{1}^{h}, u_{2}^{h}$ and $u_{3}^{h}$ are respectively first, second and third. Hence, the minimal quadratures for DIE are respectively the first, second and second order quadratures. For problem 2, the orders of $(\boldsymbol{L N})^{T} \tilde{\mathbf{s}}$ 's for $u_{1}^{h}, u_{2}^{h}$ and $u_{3}^{h}$ are respectively second, third and fourth. Hence, the minimal quadratures for DIE are respectively the second, second and third order quadratures. Tables 1 and 2 list the predicted $u^{h}$ and stress at $x=6$ for different combinations of quadrature and number of integration subdomains. Whenever the employed quadrature orders are lower than the minimal, it can be seen that the predictions are erroneous regardless of how many integration subdomains are used. The importance of DIE in securing linear and quadratic exactness is demonstrated. 
Table 1. Predicted tip deflections and stresses for problem 1 by different integration schemes.

\begin{tabular}{|c|c|c|c|c|c|c|}
\hline $\begin{array}{c}\text { trial } \\
\text { solution }\end{array}$ & $\begin{array}{c}\text { minimum } \\
\text { quadrature } \\
\text { order for DIE }\end{array}$ & $\begin{array}{c}\text { employed } \\
\text { quadrature } \\
\text { order }\end{array}$ & $\begin{array}{c}\text { no. of } \\
\text { integration } \\
\text { subdomains }\end{array}$ & $\begin{array}{c}\text { total no. of } \\
\text { integration } \\
\text { points }\end{array}$ & $\left.u^{h}\right|_{x=6}$ & $\left.\frac{d u^{h}}{d x}\right|_{x=6}$ \\
\hline$u_{1}^{h}$ & 1 & 1 & 2 & 2 & exact & exact \\
\hline \multirow{2}{*}{$u_{2}^{h}$} & 2 & 1 & 3 & 3 & 6.1875 & 1.5938 \\
& 2 & 1 & 12 & 12 & 6.0004 & 1.0180 \\
\hline \multirow{2}{*}{$u_{3}^{h}$} & 2 & 1 & 4 & 4 & 6.0417 & 1.2292 \\
& 2 & 1 & 12 & 12 & 6.0004 & 1.0180 \\
exact & exact \\
\hline
\end{tabular}

Table 2. Predicted tip deflections and stresses for problem 2 by different integration schemes.

\begin{tabular}{|c|c|c|c|c|c|c|}
\hline $\begin{array}{c}\text { trial } \\
\text { solution }\end{array}$ & $\begin{array}{c}\text { minimum } \\
\text { quadrature } \\
\text { order for DIE }\end{array}$ & $\begin{array}{l}\text { employed } \\
\text { quadrature } \\
\text { order }\end{array}$ & $\begin{array}{c}\text { no. of } \\
\text { integration } \\
\text { subdomains }\end{array}$ & $\begin{array}{l}\text { total no. of } \\
\text { integration } \\
\text { points }\end{array}$ & $\left.u^{h}\right|_{x=6}$ & $\left.\frac{d u^{h}}{d x}\right|_{x=6}$ \\
\hline$u_{1}^{h}$ & 2 & $\begin{array}{l}1 \\
1 \\
2 \\
\end{array}$ & $\begin{array}{c}2 \\
12 \\
1 \\
\end{array}$ & $\begin{array}{c}2 \\
12 \\
2 \\
\end{array}$ & $\begin{array}{l}\text { exact } \\
\text { exact } \\
\text { exact }\end{array}$ & $\begin{array}{c}-0.5000 \\
0.9685 \\
\text { exact }\end{array}$ \\
\hline$u_{2}^{h}$ & 2 & $\begin{array}{l}1 \\
1 \\
2\end{array}$ & $\begin{array}{c}3 \\
12 \\
2\end{array}$ & $\begin{array}{c}3 \\
12 \\
4 \\
\end{array}$ & $\begin{array}{c}24.7500 \\
24.0015 \\
\text { exact }\end{array}$ & $\begin{array}{c}2.8125 \\
1.0407 \\
\text { exact }\end{array}$ \\
\hline$u_{3}^{h}$ & 3 & $\begin{array}{l}1 \\
1 \\
2 \\
2 \\
3\end{array}$ & $\begin{array}{c}4 \\
12 \\
2 \\
6 \\
2\end{array}$ & $\begin{array}{c}4 \\
12 \\
4 \\
12 \\
6\end{array}$ & $\begin{array}{c}24.1667 \\
24.0015 \\
\text { exact } \\
\text { exact } \\
\text { exact }\end{array}$ & $\begin{array}{c}-5.4114 \\
0.8203 \\
-0.2500 \\
0.9954 \\
\text { exact }\end{array}$ \\
\hline
\end{tabular}

Example 2.2: To illustrate the importance of BIE, the two-dimensional elasticity problems depicted in Figure 2 are considered. The analytical solutions are:

- $\operatorname{problem} 3\left(\left.\bar{t}_{x}\right|_{x=10}=1\right): u_{x}=x, u_{y}=0, \mathrm{~s}_{x}=1, \mathrm{~s}_{y}=\mathrm{t}_{x y}=0$.

- $\operatorname{problem} 4\left(\left.\bar{t}_{x}\right|_{x=10}=y\right): u_{x}=x y, u_{y}=-x^{2} / 2, \mathrm{~s}_{x}=y, \mathrm{~s}_{y}=\mathrm{t}_{x y}=0$.

The following trial solutions are considered:

- $\quad \mathbf{u}_{1}^{h} \sim$ complete biquadratic polynomial expansion with $\left.u_{x}^{h}\right|_{x=0}=\left.u_{y}^{h}\right|_{x=y=0}=0$ constrained

- $\quad \mathbf{u}_{2}^{h} \sim$ complete bicubic polynomial expansion with $\left.u_{x}^{h}\right|_{x=0}=\left.u_{y}^{h}\right|_{x=y=0}=0$ constrained

The problem domain is partitioned into four equal-sized integration subdomains as shown in Figure 2. DIE has been secured by a sufficiently high order quadrature. Different quadratures are employed to compute the work done over the two boundary segments $-1 \leq y \leq 0$ and $0 \leq y \leq 1$ at $x=10$. For 
problem 3, the orders of $\mathbf{N}^{T} \mathbf{n} \tilde{\mathbf{s}}$ 's at $x=10$ for $\mathbf{u}_{1}^{h}$ and $\mathbf{u}_{2}^{h}$ are respectively second and third orders. Hence, the second order quadrature is the minimal quadrature for BIE. For problem 4, the orders of $\mathbf{N}^{T} \mathbf{n} \tilde{\mathbf{s}}$ 's at $x=10$ for $\mathbf{u}_{1}^{h}$ and $\mathbf{u}_{2}^{h}$ are respectively third and fourth. Hence, the second and third order quadratures are respectively the minimal quadratures for BIE. Tables 3 and 4 list the displacement at $\mathrm{A}$ and the bending stress at $\mathrm{B}$, see Figure 2. Whenever the employed quadrature orders are lower than the minimal, it can be seen that the predictions are erroneous. The importance of BIE in securing linear and quadratic exactness is demonstrated.

Table 3. Predictions for problem 3 by using different quadratures to evaluate the work done.

\begin{tabular}{|c|c|c|c|c|c|}
\hline $\begin{array}{c}\text { trial } \\
\text { solution }\end{array}$ & $\begin{array}{c}\text { minimum quadrature } \\
\text { order for BIE }\end{array}$ & $\begin{array}{c}\text { employed } \\
\text { quadrature order }\end{array}$ & $\left.u_{x}^{h}\right|_{x=10, y=0}$ & $\left.u_{y}^{h}\right|_{x=10, y=0}$ & $\left.\frac{d u_{x}^{h}}{d x}\right|_{x=5, y=1}$ \\
\hline \multirow{2}{*}{$\mathbf{u}_{1}^{h}$} & 2 & 1 & 10.0343 & exact & 0.9931 \\
& 2 & exact & exact & exact \\
& 3 & exact & exact & exact \\
\hline $\mathbf{u}_{2}^{h}$ & 2 & 2 & 10.0670 & exact & 1.0079 \\
& 3 & exact & exact & exact \\
exact & exact & exact \\
\hline
\end{tabular}

Table 4. Predictions for problem 4 by using different quadratures to evaluate the work done.

\begin{tabular}{|c|c|c|c|c|c|}
\hline trial solution & $\begin{array}{l}\text { minimum quadrature } \\
\text { order for BIE }\end{array}$ & $\begin{array}{c}\text { employed } \\
\text { quadrature order }\end{array}$ & $\left.u_{x}^{h}\right|_{x=10, y=0}$ & $\left.u_{y}^{h}\right|_{x=10, y=0}$ & $\left.\frac{d u_{x}^{h}}{d x}\right|_{x=5, y=1}$ \\
\hline $\mathbf{u}_{1}^{h}$ & 2 & $\begin{array}{l}1 \\
2 \\
3\end{array}$ & $\begin{array}{l}\text { exact } \\
\text { exact } \\
\text { exact }\end{array}$ & $\begin{array}{c}-37.5000 \\
\text { exact } \\
\text { exact }\end{array}$ & $\begin{array}{c}0.7500 \\
\text { exact } \\
\text { exact }\end{array}$ \\
\hline $\mathbf{u}_{2}^{h}$ & 3 & $\begin{array}{l}1 \\
2 \\
3\end{array}$ & $\begin{array}{l}\text { exact } \\
\text { exact } \\
\text { exact }\end{array}$ & $\begin{array}{c}-37.4059 \\
-49.9940 \\
\text { exact }\end{array}$ & $\begin{array}{c}0.7474 \\
0.9998 \\
\text { exact }\end{array}$ \\
\hline
\end{tabular}

Commonly used mesh-free approximants such as those constructed by moving least-squares and reproducing kernel particle methods [2,3] contain non-polynomial components. Therefore, BIE and DIE cannot be secured by quadatures or, to be more specific, Gaussian quadatures even for linear exactness as illustrated in reference [13]. Nevertheless, the corresponding error decreases when the quadrature order and/or the number of integration subdomains increases.

\section{DIRECT AND STABILIZED NODAL INTEGRATIONS}

An efficient way to evaluate the weak form for Galerkin mesh-free methods is nodal integration whose core idea is to use nodes as the integration sampling points and sizes of the nodal subdomains as the weight factors. To this end, (7) is revised as: 


$$
\mathbf{P}^{*}=\sum_{I=1} \mathbf{q}^{T}\left[\frac{A^{I}}{2}(\boldsymbol{L} \mathbf{N})_{I}^{T} \mathbf{C}(\boldsymbol{L} \mathbf{N})_{I} \mathbf{q}-\left\langle\mathbf{N}^{T} \overline{\mathbf{b}}\right\rangle_{\mathrm{W}_{l}}-\left\langle\mathbf{N}^{T} \overline{\mathbf{t}}\right\rangle_{\mathrm{G}_{t}^{I}}^{\#}\right]
$$

in which $I$ is the nodal index, ()$_{I}$ denotes the nodal value of the embraced term, $A^{I}$ denotes the nodal subdomain size and the superscript \# designates that the integral is computed for prescribing the boundary traction $\overline{\mathbf{t}}$. An efficient way to construct nodal subdomains is the Dirichlet tessellation which leads to the Voronoi cells [10].

In nodal integrations, the number of integration subdomains has been fixed by the number of nodes. Using the single-point quadrature which can secure the linear exactness only for linear trial solutions, the integration error for the trial solutions commonly employed in mesh-free methods appears to be significant. Though the system matrix arising from the above direct nodal integration is non-singular, its solutions are sometimes plagued by spurious oscillations which can be illustrated by the two one-dimensional problems in Example 2.1. The five-node Lagrangian interpolation is adopted as the trial solution. Oscillations of the results predicted by the direct nodal integration can be seen in Figures 3 and 4. The predictions of the stabilized conforming (SC) nodal integration will be discussed in the next section. Beissel \& Belystchko [11] stabilized the oscillation by penalizing $\Pi^{*}$ with the square of the equilibrium residue and led to:

$$
\begin{gathered}
\mathbf{P}^{* *}=\sum_{I=1}\left[\mathbf{q}^{T} \frac{A^{I}}{2}(\boldsymbol{L} \mathbf{N})_{I}^{T} \mathbf{C}(\boldsymbol{L} \mathbf{N})_{I} \mathbf{q}-\mathbf{q}^{T} A_{I}\left(\mathbf{N}^{T} \overline{\mathbf{b}}\right)_{I}-\mathbf{q}^{T}\left\langle\mathbf{N}^{T} \overline{\mathbf{t}}\right\rangle_{\mathrm{G}_{t}^{I}}^{\#}\right. \\
\left.+\mathbf{a} A^{I}\left(\boldsymbol{L}^{T} \mathbf{C}(\boldsymbol{L} \mathbf{N}) \mathbf{q}+\overline{\mathbf{b}}\right)_{I}^{T}\left(\boldsymbol{L}^{T} \mathbf{C}(\boldsymbol{L} \mathbf{N}) \mathbf{q}+\overline{\mathbf{b}}\right)_{I}\right]
\end{gathered}
$$

The same stabilized nodal integration was later adopted by Bonet \& Kulasegaram [12] who employed the smoothed particle hydrodynamics approximation. Nevertheless, the equilibrium residue involves the second order derivatives which are costly and inaccurate to be computed. For problems that do not originally exhibit the spurious modes, the stabilization term can deteriorate the solution accuracy and the penalty factor must be chosen with care [13].

\section{STABILIZED CONFORMING NODAL INTEGRATION}

By considering the equilibrium of the prescribed boundary traction and the traction derived from the domain stress/strain, Chen and his coworkers identified the integration constraint (IC) as the criteria for the Galerkin method to fulfill the linear consistency. The consistency is indeed the analogy of the satisfaction of the constant stress patch test in the finite element method. IC can be stated as: 


$$
\left\langle\boldsymbol{L} \mathbf{N}_{I}\right\rangle_{\mathrm{W}}=\left\langle\mathbf{n}^{T} \mathbf{N}_{I}\right\rangle_{G_{t}}^{\#}
$$

where $\mathbf{N}_{I}$ denotes the shape function matrix of the $I$-th node. Obviously, $\left\langle\boldsymbol{L} \mathbf{N}_{I}\right\rangle_{\mathrm{W}}$ vanishes when the support of the $I$-th node does not overlap with $\Gamma_{t}$. Unlike (12) which uses the nodal gradient to evaluate the weak form, Chen and his coworkers employed the following smoothed nodal gradient in the stabilized conforming (SC) nodal integration [13-15]:

$$
(\boldsymbol{L N})_{I} \simeq \frac{1}{A^{I}} \int_{\mathrm{W}} \boldsymbol{L} \mathbf{N} d \mathbf{W}=\frac{1}{A^{I}} \int_{\partial \mathrm{W}} \mathbf{n}^{T} \mathbf{N} d \mathbf{G} \simeq \frac{1}{A^{I}}\left\langle\mathbf{n}^{T} \mathbf{N}\right\rangle_{\partial \mathrm{W}}
$$

The second approximation " $\simeq "$ arises from the numerical integration. Thus, (12) becomes:

$$
\mathbf{P}^{@}=\mathbf{q}^{T} \sum_{I=1}\left[\frac{1}{2 A^{I}}\left\langle\mathbf{n}^{T} \mathbf{N}\right\rangle_{\partial \mathrm{W}}^{T} \mathbf{C}\left\langle\mathbf{n}^{T} \mathbf{N}\right\rangle_{\partial \mathrm{W}} \mathbf{q}-\left\langle\mathbf{N}^{T} \overline{\mathbf{b}}\right\rangle_{\mathrm{W}}-\left\langle\mathbf{N}^{T} \overline{\mathbf{t}}\right\rangle_{\mathrm{G}_{t}^{I}}^{\#}\right]
$$

and

$$
\begin{aligned}
\mathrm{dP}^{@} & =\mathrm{d} \mathbf{q}^{T} \sum_{I}\left[\frac{1}{A^{I}}\left\langle\mathbf{n}^{T} \mathbf{N}\right\rangle_{\mathrm{G}_{t}^{I}}^{T} \mathbf{C}\left\langle\mathbf{n}^{T} \mathbf{N}\right\rangle_{\partial \mathrm{W}} \mathbf{q}-\left\langle\mathbf{N}^{T} \overline{\mathbf{b}}\right\rangle_{\mathrm{W}}-\left\langle\mathbf{N}^{T} \overline{\mathbf{t}}\right\rangle_{\mathrm{G}_{t}^{I}}^{\#}\right] \\
& +\mathrm{d} \mathbf{q}^{T} \sum_{m}\left[\frac{1}{A^{m+}}\left\langle\mathbf{N}^{T} \mathbf{n}\right\rangle_{\mathrm{G}_{m}}^{m+} \mathbf{C}\left\langle\mathbf{n}^{T} \mathbf{N}\right\rangle_{\partial \mathrm{W}^{m+}}+\frac{1}{A^{m-}}\left\langle\mathbf{N}^{T} \mathbf{n}\right\rangle_{\mathrm{G}_{m}-}^{m-} \mathbf{C}\left\langle\mathbf{n}^{T} \mathbf{N}\right\rangle_{\partial \mathrm{W}^{m-}}\right] \mathbf{q}
\end{aligned}
$$

To examine the requirements for linear exactness, $\mathbf{q}, \overline{\mathbf{b}}$ and $\overline{\mathbf{t}}$ are prescribed respectively to $\tilde{\mathbf{q}}, \mathbf{0}$ and $\mathbf{n} \tilde{\mathbf{s}}$ such that $\tilde{\mathbf{u}}=\mathbf{N} \tilde{\mathbf{q}}$ is an arbitrary linear displacement field and $\tilde{\mathbf{s}}=\mathbf{C}(\boldsymbol{L} \mathbf{N}) \tilde{\mathbf{q}}$ is the corresponding constant stress field. Assuming

$$
\tilde{\mathbf{s}}=\frac{1}{A^{I}} \mathbf{C}\left\langle\mathbf{n}^{T} \mathbf{N}\right\rangle_{\partial \mathrm{W}} \tilde{\mathbf{q}}=\frac{1}{A^{I}} \mathbf{C}\left\langle\mathbf{n}^{T} \tilde{\mathbf{u}}\right\rangle_{\partial \mathrm{W}}
$$

then

$$
\left.\mathrm{dP}^{@}\right|_{\mathbf{q}=\overline{\mathbf{q}}, \overline{\mathbf{b}}=\mathbf{0}, \overline{\mathbf{t}}=\mathbf{n} \tilde{\mathbf{s}}}=\mathrm{d} \mathbf{q}^{T} \sum_{I}\left[\left\langle\mathbf{N}^{T} \mathbf{n}\right\rangle_{\mathrm{G}_{t}^{l}}-\left\langle\mathbf{N}^{T} \mathbf{n}\right\rangle_{\mathrm{G}_{t}^{l}}^{\#}\right] \tilde{\mathbf{s}}+\mathrm{d} \mathbf{q}^{T} \sum_{m}\left[\left\langle\mathbf{N}^{T} \mathbf{n}\right\rangle_{\mathrm{G}_{m}}^{m+}+\left\langle\mathbf{N}^{T} \mathbf{n}\right\rangle_{\mathrm{G}_{m}}^{m-}\right] \tilde{\mathbf{s}}
$$

Should the system matrix be non-singular, the sufficient conditions for $\mathbf{q}=\tilde{\mathbf{q}}, \overline{\mathbf{b}}=\mathbf{0}$ and $\overline{\mathbf{t}}=\mathbf{n} \tilde{\mathbf{s}}$ being the unique solution of the above weak form are the following boundary integration consistency (BIC) requirements:

$$
\left\langle\mathbf{N}^{T} \mathbf{n}\right\rangle_{\mathrm{G}_{t}^{I}}=\left\langle\mathbf{N}^{T} \mathbf{n}\right\rangle_{\mathrm{G}_{t}^{I}}^{*},\left\langle\mathbf{N}^{T} \mathbf{n}\right\rangle_{\mathrm{G}_{m}}^{m+}+\left\langle\mathbf{N}^{T} \mathbf{n}\right\rangle_{\mathrm{G}_{m}}^{m-}=0
$$

If non-polynomial terms are involved in the trial solution, it will not be possible to evaluate $\left\langle\mathbf{N}^{T} \mathbf{n}\right\rangle$ exactly by mid-point, trapezoidal or Gaussian rules. Fortunately, when the same integration rule is applied to evaluate the two integrands in the first BIC requirement and the two integrands in the second BIC requirement, pairwise cancellation of traction similar to that of the free formulation 
[16-18] will occur.

Unlike BIE, exactness is not posed by BIC. BIC is far less demanding and can easily be satisfied, for instances, by using the same integration rule to evaluate all integrands over the same boundary segment. The practice is not only natural but is also the one employed in SC nodal integration [1315]. This completes the proof for the linear exactness of the SC nodal integration. By merging and assembling the two BIC requirements, one can obtain:

$$
\left\langle\mathbf{N}^{T} \mathbf{n}\right\rangle_{\partial \mathrm{W}}=\left\langle\mathbf{N}^{T} \mathbf{n}\right\rangle_{\mathrm{G}_{t}}^{*}
$$

which is equivalent to the IC in (14) posed for linear exactness in reference [13]. Hence, this paragraph presents not only an explicit proof but also a more "microscopic" analysis for SC nodal integration.

The two problems in Example 2.1 are repeated by using the five-node interpolation and SC nodal integration. Linear exactness is yielded as shown in Figure 3. To compute the system matrix of onedimensional problems, SC nodal integration only needs to compute the trial solution at the node-tonode mid-points and the two boundary nodes, see Figure 5. Hence, it is more efficient to account for the body force by using the trapezoidal rule than the rectangular rule. The two practices lead to respectively SC(trap.) and SC(rect.) in Figure 4. The former yields quadratic exactness in this illustration, which employs a polynomial trial solution, and the latter is less accurate.

\section{VARIATIONAL BASIS OF SC NODAL INTEGRATION}

Strain smoothing in SC nodal integration was introduced into the Galerkin mesh-free methods through the assumed strain method [13]. In this section, the basis of SC nodal integration and thus the strain smoothing will be provided by a classical variational functional for domain decomposition. By taking stress and displacement as the independent field variables as well as treating e.b.c. and displacement compatibility as prerequisites, the strong form of the problem can be summarized as:

- $\quad$ stress-displacement relation: $\mathbf{s}=\mathbf{C}(\boldsymbol{L} \mathbf{u})$

$$
\begin{aligned}
& \text { in all } \Omega^{I \text { 's }} \\
& \text { - } \quad \text { stress equilibrium condition: } \boldsymbol{L}^{T} \mathbf{S}+\overline{\mathbf{b}}=\mathbf{0} \quad \text { in all } \Omega^{I \text {, } \mathrm{s}} \\
& \text { - n.b.c.: } \mathbf{n s}=\overline{\mathbf{t}} \quad \text { on all } \mathrm{G}_{t}^{I}
\end{aligned}
$$$$
\text { - traction reciprocity: }(\mathbf{n s})^{m+}+(\mathbf{n s})^{m-}=\mathbf{0} \quad \text { on all } \mathbf{G}_{m} \text { 's }
$$

The following is the domain-decomposed version of the well-known Hellinger-Reissner functional 
[18-21]:

$$
\mathbf{P}_{H R}=\sum_{I}\left(\int_{\mathrm{W}}\left[-\frac{1}{2} \mathbf{s}^{T} \mathbf{C}^{-1} \mathbf{S}+\mathbf{S}^{T}(\boldsymbol{L} \mathbf{u})-\mathbf{u}^{T} \overline{\mathbf{b}}\right] d \mathbf{W}-\int_{\mathrm{G}_{l}^{I}} \mathbf{u}^{T} \overline{\mathbf{t}} d \mathrm{G}\right)
$$

By virtue of the divergence theorem,

$$
\begin{aligned}
\mathrm{dP}_{H R}= & \sum_{I}\left(\int_{\mathbf{W}}\left[\mathrm{d} \mathbf{s}^{T}\left(-\mathbf{C}^{-1} \mathbf{s}+\boldsymbol{L} \mathbf{u}\right)-\left(\boldsymbol{L}^{T} \mathbf{s}+\overline{\mathbf{b}}\right)^{T} \mathrm{~d} \mathbf{u}\right] d \mathbf{W}+\int_{\mathrm{G}_{t}^{I}} \mathrm{~d} \mathbf{u}^{T}(\mathbf{n s}-\overline{\mathbf{t}}) d \mathbf{G}\right) \\
& +\sum_{m} \int_{\mathrm{G}_{m}} \mathrm{~d} \mathbf{u}^{T}\left[(\mathbf{n s})^{m+}+(\mathbf{n s})^{m-}\right] d \mathbf{G}
\end{aligned}
$$

it can be seen that (22) to (25) are enforced by the stationary nature of $\Pi_{H R}$. For $\boldsymbol{\sigma}$ satisfying the homogeneous equilibrium condition, i.e. $\boldsymbol{L}^{T} \mathbf{s}=\mathbf{0}$,

$$
\mathbf{P}_{H R}=\sum_{I}\left(\int_{W}\left[-\frac{1}{2} \mathbf{s}^{T} \mathbf{C}^{-1} \mathbf{S}-\mathbf{u}^{T} \overline{\mathbf{b}}\right] d \mathbf{W}+\int_{\partial \mathrm{W}}(\mathbf{n s})^{T} \mathbf{u} d \mathbf{G}-\int_{\mathrm{G}_{t}^{I}} \mathbf{u}^{T} \overline{\mathbf{t}} d \mathbf{G}\right)
$$

In hybrid element formulation, the use of homogeneous equilibrating stress enables the element stiffness matrix to be constructed by defining the displacement along the element boundary only. This is particularly advantageous when a compatible displacement cannot be constructed inside the element subdomain [19-21]. With the homogeneous equilibrating trial stress expressed as:

$$
\mathbf{S}=\mathbf{P b}^{I}
$$

in which $\mathbf{P}$ is the stress shape function matrix and $\boldsymbol{\beta}^{I}$ is the vector of coefficients for the $I$-th nodal subdomain, (28) becomes:

$$
\mathbf{P}_{H R}=\sum_{I}\left(-\frac{1}{2}\left(\mathbf{b}^{I}\right)^{T} \int_{W} \mathbf{P}^{T} \mathbf{C}^{-1} \mathbf{P} d \mathbf{W} \cdot \mathbf{b}^{I}+\left(\mathbf{b}^{I}\right)^{T} \int_{\partial W} \mathbf{P}^{T} \mathbf{n}^{T} \mathbf{N} d \mathbf{G} \cdot \mathbf{q}-\mathbf{q}^{T} \int_{W} \mathbf{N}^{T} \overline{\mathbf{b}} d \mathbf{W}-\mathbf{q}^{T} \int_{\mathrm{G}_{t}^{I}} \mathbf{N}^{T} \overline{\mathbf{t}} d \mathbf{G}\right)
$$

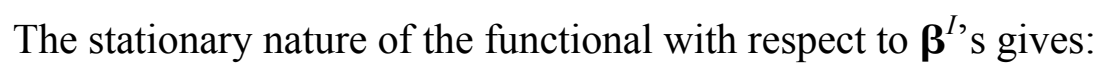

$$
\int_{W} \mathbf{P}^{T} \mathbf{C}^{-1} \mathbf{P} d \mathbf{W} \cdot \mathbf{b}^{I}=\int_{\partial \mathrm{W}} \mathbf{P}^{T} \mathbf{n}^{T} \mathbf{N} d \mathbf{G} \cdot \mathbf{q}
$$

with which

$$
\mathbf{P}_{H R}=\mathbf{q}^{T} \sum_{I}\left(\frac{1}{2}\left(\int_{\partial \mathbf{W}} \mathbf{P}^{T} \mathbf{n}^{T} \mathbf{N} d \mathbf{G}\right)^{T}\left(\int_{W} \mathbf{P}^{T} \mathbf{C}^{-1} \mathbf{P} d \mathbf{W}\right)^{-1}\left(\int_{\partial \mathbf{W}} \mathbf{P}^{T} \mathbf{n}^{T} \mathbf{N} d \mathbf{G}\right) \mathbf{q}-\int_{\mathbf{W}} \mathbf{N}^{T} \overline{\mathbf{b}} d \mathbf{W}-\int_{\mathrm{G}_{t}^{I}} \mathbf{N}^{T} \overline{\mathbf{t}} d \mathbf{G}\right)
$$

When $\mathbf{P}$ is chosen to be the identity matrix and numerical integration is invoked, the functional will be: 


$$
\mathbf{P}_{H R}^{\#}=\mathbf{q}^{T} \sum_{I=1}\left[\frac{1}{2 A^{I}}\left\langle\mathbf{n}^{T} \mathbf{N}\right\rangle_{\partial \mathrm{W}}^{T} \mathbf{C}\left\langle\mathbf{n}^{T} \mathbf{N}\right\rangle_{\partial \mathrm{W}} \mathbf{q}-\left\langle\mathbf{N}^{T} \overline{\mathbf{b}}\right\rangle_{\partial \mathrm{W}}-\left\langle\mathbf{N}^{T} \overline{\mathbf{t}}\right\rangle_{\mathrm{G}_{t}^{I}}^{*}\right]
$$

which is identical to $\Pi^{\circledR}$ in (16). In this light, the SC nodal integration is justified variationally by the classical Hellinger-Reissner principle.

\section{NUMERICAL EXAMPLES}

As a large number of one-dimensional and two-dimensional linear and nonlinear problems have been studied by SC nodal integration using the reproducing kernel particle approximants [13-15], only a few examples will be shown here to contrast the direct and stabilized conforming nodal integrations. Moreover, the least-squares (MLS) approximants [2] will be employed. For completeness, a brief introduction to the MLS method is presented in Appendix A. The weight function is taken to be the $C^{2}$ quartic spline function. To facilitate the subsequent discussion, the following abbreviations are defined:

- $\mathrm{Nn}-$ the number of uniformly distributed nodes in the problem

- $\mathrm{Np}$ - the order of the MLS basis functions (1 for linear basis, 2 for quadratic basis)

- $\mathrm{Ln}$ - nodal spacing

Example 6.1 1D problem with zero body force, see Example 2.1: Problem 1 is repeated with $\mathrm{Nn}=$ $7, \mathrm{~Np}=2$ and $\mathrm{R}=3 \mathrm{Ln}$. It can be seen in Figure 6 that SC nodal integration yields the exact solution whereas direct nodal integration deviates slightly from the exact solution. Oscillation of results predicted by direct nodal integration can be seen but is not obvious.

Example 6.2 1D problem with constant body force, see Example 2.1: Problem 2 is repeated with $\mathrm{Nn}=7, \mathrm{~Np}=2$ and $\mathrm{R}=3 \mathrm{Ln}$. Slight oscillation of results predicted by direct nodal integration can be noted in Figure 7. For both kinds of nodal integrations, the use of the trapezoidal rule to account for the body force yields more accurate results than the use of the rectangular rule. Though SC(trap.) graphically overlaps the exact solution, they differ numerically. In other words, the present SC(trap.) does not attain quadratic exactness as the one that employs purely polynomial terms in the trial solutions, see Figure 4.

Example 6.3 1D problem with linear body force: The following problem was considered by Beissel \& Belystchko [11] and is excellent for demonstrating the spurious oscillation: 


$$
\frac{d^{2} u}{d^{2} x}+\frac{x}{10}=0 \text { for } 0 \leq \mathrm{x} \leq 10,\left.u\right|_{x=0}=\left.u\right|_{x=10}=0 \Rightarrow u=\frac{5 x}{3}\left(1-\frac{x^{2}}{100}\right)
$$

By using $\mathrm{Nn}=11, \mathrm{R}=3 \mathrm{Ln}$ and $\mathrm{Np}=2$, Figure 8 shows again that the use of the trapezoidal rule to account for the body force yields marginally more accurate results than the use of the rectangular rule in accounting for the body force. It can be seen that SC(trap.) graphically overlaps with the exact solution. Spurious oscillation is obvious in the predictions of direct nodal integration. Since the rectangular and trapezoidal rules are most natural to evaluate the body force term in respectively the direct and the SC nodal integrations, they will be adopted accordingly in the remaining studies. Figures 9 and 10 examine the predictions of direct nodal integration by using different $\mathrm{R}, \mathrm{Np}$ and $\mathrm{Nn}$. It is worthwhile to note that the results actually diverge from the exact solution as the number of nodes increase. On the other hand, the use of higher $\mathrm{Nn}$ in SC nodal integration yields progressively more accurate results as seen in Figures 11 and 12.

Regarding the source of the strong spurious oscillation in the direct nodal integration, it is interesting to note that both the nodal gradient (used in direct nodal integration) and the smoothed nodal gradient (used in SC nodal integration) are independent of the nodal parameters at the same node for the node whose support does not reach the boundary. Hence, this independence does not appear to be the cause of the oscillation. A more convincing cause is probably the poor enforcement of the traction reciprocity in the direct nodal integration.

Example 6.4 A simply supported beam subjected to midspan force: For a thin beam with its centroidal axis bounded by $x \square\left[x_{S}, x_{T}\right]$ coincident with the $x$-axis, the inplane strain is $\mathrm{e}_{x x}=-z w,_{x x}$ and the strain energy is

where

$$
U=\frac{1}{2} \int_{x_{S}}^{x_{T}} \int_{-h / 2}^{+h / 2} E \mathrm{e}_{x x}^{2} d z d x=\frac{1}{2} \int_{x_{S}}^{x_{T}} \int_{h / 2}^{+h / 2} z^{2} E w_{x x}^{2} d z d x=\frac{1}{2} \int_{x_{S}}^{x_{T}} E^{b} w_{x x}^{2} d x
$$

$$
h \text { is the beam thickness and } E^{b}=\int_{-h / 2}^{+h / 2} z^{2} E d x .
$$

Using the SC nodal integration, the strain energy in a nodal subdomain $x \in\left[x_{S}^{I}, x_{T}^{I}\right]$ becomes

$$
U^{I}=\frac{E^{b}}{2}\left(\bar{w}_{x x}^{I}\right)^{2}
$$

where 


$$
\bar{w}_{, x x}^{I}=\int_{x_{S}^{I}}^{x_{T}^{I}} w,_{x x} d x=\left.\left(w,{ }_{x}\right)\right|_{x=x_{T}^{I}}-\left.\left(w,_{x}\right)\right|_{x=x_{S}^{I}} .
$$

Figure 13 shows a simply supported beam with a mid-span force $P$ acting. The thin beam solution for the mid-span deflection is $\left(P l^{3}\right) /\left(4 E h^{3}\right)$ where $l$ is beam length and $h$ is the beam thickness. After normalized by the thin beam solution, the predicted mid-span deflections are plotted against $\mathrm{R} / \mathrm{Ln}$ in Figure 14 for quadratic basis (i.e. $\mathrm{Np}=2$ ). The results of the $\mathrm{SC}$ nodal integration and the ones obtained by direct integration of the strain energy with the one-, two- and three-point quadratures to each node-to-node subdomain are shown. The accuracy of SC nodal integration is more accurate than, close to and less accurate than that of the one-, two- and threepoint quadratures, respectively. However, the SC nodal integration is more economic than all the quadrature scheme due to the reduction of derivative order.

Example 6.5 A thin square plate subjected to central point force: For a plate with its mid-plane $A$ at $z=0$, the vector of inplane strain components is: $\mathbf{e}=z \mathbf{e}^{b}$ where $\mathbf{e}^{b}=-\left\{w,{ }_{x x}, w,_{y y}, 2 w,_{x y}\right\}^{T}$ is the vector of bending strain components. The strain energy is:

$$
U=\frac{1}{2} \int_{A}^{+h / 2} \int_{-h / 2}^{2} z^{2}\left(\mathbf{e}^{b}\right)^{T} \mathbf{C}\left(\mathbf{e}^{b}\right) d z d A=\frac{1}{2} \int_{A}\left(\mathbf{e}^{b}\right)^{T} \mathbf{C}^{b} \mathbf{e}^{b} d A
$$

where $\mathbf{C}$ is the elasticity matrix with the plane stress condition incorporated along the $z$-direction and

$$
\mathbf{C}^{b}=\int_{-h / 2}^{+h / 2} z^{2} \mathbf{C} d z
$$

Using the SC nodal integration, the strain energy in the nodal subdomain $A^{I}$ is

$$
U^{I}=\frac{A^{I}}{2}\left(\overline{\mathbf{e}}^{b I}\right)^{T} \mathbf{C}^{b}\left(\overline{\mathbf{e}}^{b I}\right)
$$

where

$$
\overline{\mathbf{e}}^{b I}=\int_{A} \mathbf{e}^{b} d A=-\int_{A}\left\{w,_{x x}, w,_{y y}, 2 w,_{x y}\right\}^{T} d A=-\oint_{\partial A}\left\{n_{x} w,_{x}, n_{y} w,_{y}, n_{x} w,_{y}+n_{y} w,_{x}\right\} d s
$$

and $\left\{n_{x}, n_{y}\right\}^{T}$ is the unit normal to $\square A$, namely, the boundary of $A$.

A simply-supported square plate of side length $a$ subjected to a central point load $P$ is considered. The converged Navier solution for the central deflection is: $0.0116 \mathrm{~Pa}^{2} / D$ where $D$ is the flexural rigidity of the plate. Again, the quadratic basis is used in MLS approximation. The plate is modeled by $11 \times 11$ uniformly, 17x17 uniformly, and 17x17 non-uniformly distributed nodes. The 
Voronoi cells for the $17 \times 17$ non-uniformly distributed nodes are shown in Figure 15. Figure 16 shows the normalized central deflections predicted by the SC nodal integration and by applying the third order Gaussian rule to the square subdomains with the uniformly distributed nodes being their corners. Despite of the shape contrast in the computational effort, it can be seen that the two integration schemes yield close accuracy. The predictions of the SC nodal integration in the $17 \times 17$ uniformly and non-uniformly distributed nodes are very close. This is probably due to the inherent insensitivity of the MLS toward nodal uniformity. The predictions from the first and second order Gaussian rules are also computed but they are all worse than that of the third order Gaussian rules.

Example 6.6 A thin circular arc subjected to end shear force: A brief account on computing the physical membrane and bending strains for thin shells with position vector $\mathbf{x}=\mathbf{x}_{o}(\mathbf{x}, \mathrm{h})+\mathbf{Z} \mathbf{x}_{n}(\mathbf{x}, \mathrm{h})$ and displacement vector $\mathbf{u}=\mathbf{u}_{o}(\mathbf{x}, \mathrm{h})+\mathbf{Z} \mathbf{u}_{n}(\mathbf{x}, \mathrm{h})$ where $\times$ and $\%$ are parametric coordinates and $=$ is the transverse coordinate along the director $\mathbf{u}_{n}$ can be found in Appendix B. For a circular arc with mean radius $R, \times=\theta \square\left[{ }_{S},{ }^{\circledR} T\right], \%=z, \mathbf{u}_{o}=\{u, v, 0\}^{T}$ and $\mathbf{x}_{o}=\left\{R \cos \AA, R \sin \AA^{\circledR}, 0\right\}^{T}$. With $\mathbf{e}_{1}=\left\{-\sin { }^{\circledR}, \cos \AA, 0\right\}^{T}$ which is tangential to $\mathbf{x}_{0}$, , the membrane and bending strains can be derived to be:

$$
\mathrm{e}^{m}=\mathrm{e}_{11}^{m}=\frac{1}{R}\left(-u, \sin \mathrm{q}+v,{ }_{\mathrm{q}} \cos \mathrm{q}\right), \mathrm{e}^{b}=\mathrm{e}_{11}^{b}=\frac{1}{R^{2}} \mathrm{e}_{\mathrm{qq}}^{b}=\frac{-1}{R^{2}}\left(u,{ }_{\mathrm{qq}} \cos \mathrm{q}+v,{ }_{\mathrm{qq}} \sin \mathrm{q}\right)
$$

and strain energy is:

$$
U=\frac{R}{2} \int_{\mathrm{q}_{S}}^{\mathrm{q}_{T}} \int_{h / 2}^{+h / 2} E\left[\left(\mathrm{e}_{11}^{m}\right)^{2}+\mathrm{z}\left(\mathrm{e}_{11}^{b}\right)^{2}\right] d \mathrm{z} d \mathbf{q}=\frac{R}{2} \int_{\mathrm{q}_{s}}^{\mathrm{q}_{T}}\left[E^{m}\left(\mathrm{e}_{11}^{m}\right)^{2}+E^{b}\left(\mathrm{e}_{11}^{b}\right)^{2}\right] d \mathbf{q}
$$

where

$$
E \text { is the elastic modulus, } E^{m}=\int_{-h / 2}^{+h / 2} E d \mathrm{z} \text { and } E^{b}=\int_{-h / 2}^{+h / 2} \mathrm{z}^{2} E d \mathrm{z} .
$$

Using the notion of SC nodal integration to smooth the displacement derivatives, the strain energy for the nodal subdomain defined at $\mathrm{q}=\mathrm{q}^{I}$ and bounded by $\mathrm{q}_{S}^{I}$ and $\mathrm{q}_{T}^{I}$ is:

where

$$
U^{I}=\frac{R}{2}\left[E^{m}\left(\overline{\mathrm{e}}^{m I}\right)^{2}+E^{b}\left(\overline{\mathrm{e}}^{b I}\right)^{2}\right]
$$

$$
\begin{aligned}
& \overline{\mathrm{e}}^{m I}=-\frac{1}{R}\left(\left.u\right|_{\mathbf{q}=\mathbf{q}_{T}^{I}}-\left.u\right|_{\mathbf{q}=\mathbf{q}_{S}^{I}}\right) \sin \mathbf{q}^{I}+\frac{1}{R}\left(\left.v\right|_{\mathbf{q}=\mathbf{q}_{T}^{I}}-\left.v\right|_{\mathbf{q}=\mathbf{q}_{S}^{I}}\right) \cos \mathbf{q}^{I}, \\
& \overline{\mathrm{e}}^{b I}=-\frac{1}{R^{2}}\left[\left(u,\left.\right|_{\mathrm{q}=\mathbf{q}_{T}^{I}}-\left.u\right|_{\mathbf{q}=\mathbf{q}_{S}^{I}}\right) \cos \mathbf{q}^{I}+\left(v,\left.\right|_{\mathbf{q}=\mathbf{q}_{T}^{I}}-\left.v\right|_{\mathbf{q}=\mathbf{q}_{S}^{I}}\right) \sin \mathbf{q}^{I}\right] \text {. }
\end{aligned}
$$

Figure 15 shows a simply supported semi-circular arc subjected a mid-span force $P$. The 
analytical thin arc solution, which account for the bending and membrane deformation, for the deflection under $P$ is derived to be:

$$
\frac{P}{E} \frac{\mathrm{r}}{h}\left[\frac{\mathrm{p}}{8}+\frac{3(3 \mathrm{p}-8)}{2}\left(\frac{\mathrm{r}}{h}\right)^{2}\right]
$$

where ${ }^{\odot}$ and $h$ are respectively the mean radius and thickness of the arc. Same as the last two examples, only quadratic basis is employed in the MLS approximation. Figures 16a and 16b show the normalized deflections for $\odot / h=50$ by using 15 and 31 uniformly distributed nodes along the arc, respectively. The weak form is evaluated by the SC nodal integration and the ones obtained by direct integration of the strain energy with the one-, two- and three-point quadratures to each nodeto-node subdomain are shown. It can be seen that all integration schemes yield more accurate results when more nodes are employed. Among them, the SC nodal integration is most accurate whereas the results yielded by the one- and three-point quadratures are too "soft" and too "stiff", respectively. By fixing the number of nodes to 31, Figures 16c shows the normalized deflections for $\odot / h=500$, respectively. By comparing Figures $16 \mathrm{~b}$ and $16 \mathrm{c}$, it can be seen that the normalized results of the SC nodal integration and the one-point quadrature are un-affected by the aspect ratio $\odot / h$. On the other hand, the normalized results of the two- and three-point quadratures drop. This is an indication that the membrane strain/energy is excessively sampled. In this constrained problem (with zero membrane strain), the most accurate integration scheme is the SC nodal integration. 


\section{CLOSURE}

Galerkin mesh-free methods are examined from a domain decomposition perspective. Presuming the analytical solution is admissible in the trial solution, requirements on integration exactness are identified for the strict satisfaction of traction reciprocity and natural boundary condition in the weak form. Mesh-free approximants often contain non-polynomial terms. When they are employed in the trial solution, the corresponding weak form cannot be exactly integrated by Gaussian quadratures. Consequently, they fail to reproduce any order of exactness. Recently, stabilized conforming nodal integration for Galerkin mesh-free methods was proposed and illustrated to be linearly exact. Provided that the same numerical integration rule is applied to integrands over the same boundary segments, this paper presents a direct proof of the linear exactness of the integration. It also shows that the integration method can be formulated by the Hellinger-Reissner Principle and thus justified in light of the classical variational sense. Applications of the method to straight beam, plate and curved beam problems are presented. Overall speaking, the SC integration offer the best combination of accuracy of computational efficiency.

Acknowledgments - The financial support of the University of Hong Kong in the form of a Small Project Funding Program is gratefully acknowledged. 


\section{REFERENCES}

1. Nayroles B, Touzot G, Villon P. Generalizing the finite element method: diffuse approximation and diffuse elements. Computational Mechanics, 10: 307-318 (1992)

2. Belytschko T, Lu YY, Gu L. Element-free Galerkin methods. Inter.J.Numer.Methods Engrg., 37: 229-256. (1994)

3. Liu WK, Jun S, Zhang YF. Reproducing kernel particle methods. Inter.J.Numer.Methods Engrg., 20: 1081-1106 (1995)

4. Duarte CAM, Oden JT. A hp adaptive method using clouds. Computer Methods Appl.Mech. Engrg., 139: 237-262 (1996)

5. Melenk JM., Babuska I. The partition of unity finite element method: basic theory and applications. Computer Methods Applied Mech. \& Engrg, 139: 289-314 (1996)

6. Atluri SN, Zhu T. A new meshless Local Petrov-Galerkin (MLPG) method. Computational Mechanics, 22: 117-127 (1998)

7. Monaghan JJ. An introduction to SPH. Computer Physics Commun., 48: 89-96 (1988)

8. Randles PW, Libersky LD. Smoothed particle hydrodynamics: some recent improvements and applications. Computer Methods Applied Mech. \& Engrg, 139: 375-408 (1996)

9. Park SH, Youn SK. The least-squares meshfree method. Inter.J.Numer.Methods Engrg., 52: 997-1012 (2001)

10. Bowyer A. Computing Dirichlet tessellations. Comp. J., 24: 162-166 (1981)

11. Beissel S, Belytschko T. Nodal integration of the element-free Galerkin method. Computer Methods Applied Mech. \& Engrg., 139: 49-74 (1996)

12. Bonet J, Kulasegaram S. Correction and stabilization of smooth particle hydrodynamics methods with applications in metal forming simulation. Inter.J.Numer.Methods Engrg., 47: 1189-1214 (1999)

13. Chen JS, Wu CT, Yoon S, You Y. A stabilized conforming nodal integration for Galerkin meshFree methods. Inter.J.Numer.Methods Engrg., 50: 435-466 (2001)

14. Chen JS, Yoon S, Wu CT. Non-linear version of stabilized conforming nodal integration for Galerkin mesh-free methods. Inter.J.Numer.Methods Engrg., 53: 2587-2615 (2002)

15. You Y, Chen JS, Voth TE. Characteristics of semi- and full discretization of stabilized Galerkin meshfree method. Finite Elements in Analysis \& Design, 38: 999-1012 (2002)

16. Bergan PG. Finite elements based on energy orthogonal functions. Inter.J.Numer.Methods. Engrg., 15: 1541-1555 (1980)

17. Felippa CA, Bergan PG. A triangular element based on an energy-orthogonal free formulation. Computer Methods Appl.Mech.Engrg., 61: 129-160 (1987)

18. Sze KY. Efficient formulation of robust hybrid elements using orthogonal stress/strain interpolants and admissible matrix formulation. Inter.J.Numer.Methods Engrg., 35: 1-20 (1992)

19. Pian THH, Tong P. Basis of finite elements for solids continua. Inter.J.Numer.Methods Engrg., 1: 3-28 (1969)

20. Pian THH. State-of-the-Art development of hybrid/mixed finite element method. Finite Elements in Analysis \& Design, 21: 5-20 (1995)

21. Sze KY, Chow CL. An efficient hybrid stress quadrilateral Kirchhoff plate bending element. Inter.J.Numer.Methods Engrg., 32: 149-169 (1991) 


\section{APPENDIX A. MOVING LEAST SQUARES METHOD}

Trial solutions in the Galerkin mesh-free methods can be constructed by different methods. Among them, moving least square (MLS) and reproducing kernel particle approximations are probably the most popular ones [2,3]. Provided the same monomial basis is employed, both approximations will yield the same trial solution. In the MLS, an approximation $f(\mathbf{x})$ localized at $\mathbf{x}$ is expressed as the following inner product:

$$
f(\mathbf{x})=\mathbf{p}^{T}(\mathbf{x}) \cdot \mathbf{a}(\mathbf{x})
$$

where $\mathbf{p}(\mathbf{x})$ is a vector comprising a set of monomial basis functions and $\mathbf{a}(\mathbf{x})$ is the associated vector of coefficients. In two-dimensions, $\mathbf{p}^{T}(\mathbf{x})=\left[1, x_{1}, x_{2}\right]$ and $\mathbf{p}^{T}(\mathbf{x})=\left[1, x_{1}, x_{2}, x_{1}^{2}, x_{1} x_{2}, x_{2}^{2}\right]$ for the linear and quadratic bases, respectively. The coefficient vector is determined by minimizing the following term with respect to the coefficient vector:

$$
J(\mathbf{x})=\sum_{I=1}^{N} w_{I}(\mathbf{x})\left(\mathbf{p}^{T}\left(\mathbf{x}_{I}\right) \cdot \mathbf{a}(\mathbf{x})-f_{I}\right)^{2}
$$

in which $\mathbf{x}_{I}, w_{I}(\mathbf{x})=w\left(\mathbf{x}-\mathbf{x}_{I}\right)$ and $f_{I}$ are the coordinate vector, weight function and the datum of the $I-$ th node, respectively. Moreover, $N$ is the number of nodes whose weight functions are non-zero at $\mathbf{x}$. The minimization yields:

$$
\mathbf{a}(\mathbf{x})=\mathbf{A}^{-1}(\mathbf{x}) \cdot \mathbf{D}(\mathbf{x}) \cdot \underline{\mathbf{f}}
$$

where

$$
\mathbf{A}(\mathbf{x})=\sum_{I=1}^{N} w_{I}(\mathbf{x}) \cdot \mathbf{p}\left(\mathbf{x}_{I}\right) \cdot \mathbf{p}^{T}\left(\mathbf{x}_{I}\right), \mathbf{D}(\mathbf{x})=\left[w_{1}(\mathbf{x}) \mathbf{p}\left(\mathbf{x}_{1}\right), \ldots, w_{N}(\mathbf{x}) \mathbf{p}\left(\mathbf{x}_{N}\right)\right], \underline{\mathbf{f}}=\left[f_{I}, \ldots, f_{N}\right]^{T}
$$

Back substitution of (a.iii) into (a.i) gives:

$$
f(\mathbf{x})=\mathbf{f}(\mathbf{x}) \cdot \underline{\mathbf{f}}
$$

in which $\mathbf{f}(x)=\mathbf{p}^{T}(\mathbf{x}) \cdot \mathbf{A}^{-1}(\mathbf{x}) \cdot \mathbf{D}(\mathbf{x})$ is the shape function matrix. As MLS is non-interpolatory,

$$
f\left(\mathbf{x}_{I}\right)=\mathbf{f}\left(\mathbf{x}_{I}\right) \cdot \underline{\mathbf{f}} \neq f_{I}
$$

in general. The first derivative of $f(\mathbf{x})$ with respect to $x_{i}$ can be derived by chain rule:

$$
f_{,_{i}}=\mathbf{f},,_{i} \underline{\mathbf{f}}=\left[\mathbf{p},{ }_{i}^{T} \mathbf{A}^{-1} \mathbf{D}+\mathbf{p}^{T} \mathbf{A}^{-1}\left(\mathbf{D},{ }_{i}-\mathbf{A},{ }_{i} \mathbf{A}^{-1} \mathbf{D}\right)\right] \cdot \underline{\mathbf{f}}
$$

Similarly, higher order derivatives can be obtained. It can be seen that calculating derivatives and, especially, calculating higher order derivatives for MLS approximation is rather costly. 
The continuity order of $f(\mathbf{x})$ is the same as the weight function $w(\mathbf{x})$. Commonly-used weight functions include exponential, Gaussian and spline functions which are at least $\mathrm{C}^{1}$. For instance, a widely-used spline is:

$$
w(r)=\left\{\begin{array}{cc}
1-6(r / R)^{2}+8(r / R)^{3}-3(r / R)^{4} & \text { for } r \leq 1 \\
0 & \text { otherwise }
\end{array}\right.
$$

in which $r=\left\|\mathbf{x}-\mathbf{x}_{I}\right\|$ is the radial distance and $R$ is the support radius. It can be checked that the function is $\mathrm{C}^{2}$. 


\section{APPENDIX B. STRAINS IN THIN SHELLS}

Let $\times$ and $c \%$ be parametric coordinates of the mid-surface $\mathbf{x}_{o}$ of the shell and $=\square[-h / 2,+h / 2]$ be the physical transverse coordinate, the position and displacement vectors of the shell can be expressed as:

$$
\mathbf{x}=\mathbf{x}_{o}(\mathbf{x}, \mathrm{h})+\mathbf{Z} \mathbf{x}_{n}(\mathbf{x}, \mathrm{h}), \quad \mathbf{u}=\mathbf{u}_{o}(\mathbf{x}, \mathrm{h})+\mathbf{Z} \mathbf{u}_{n}(\mathbf{x}, \mathrm{h})
$$

where $\mathbf{x}_{n}=\mathbf{x}_{o, \mathrm{x}} \times \mathbf{x}_{o, \mathrm{~h}} / j$ and $j=\left\|\mathbf{x}_{o, \mathrm{x}} \times \mathbf{x}_{o, \mathrm{~h}}\right\|$. The linear covariant inplane strain $\varepsilon_{i j}$ and transverse shear strain $\varepsilon_{\zeta i}$ are

where

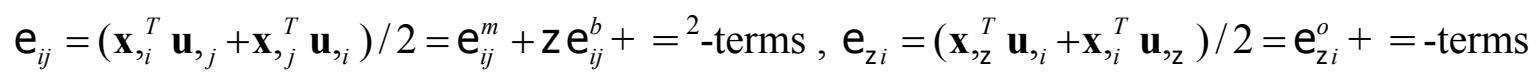

$i, j=\times$ and $\%, \mathrm{e}_{z i}^{o}=\mathbf{x}_{n}^{T} \mathbf{u}_{o, i}+\mathbf{x}_{o, i}^{T} \mathbf{u}_{n}, \mathrm{e}_{i j}^{m}=\left(\mathbf{x}_{o, i}^{T} \mathbf{u}_{o, j}+\mathbf{x}_{o, j}^{T} \mathbf{u}_{o, i}\right) / 2$ is the membrane strain, $\mathrm{e}_{i j}^{b}=\left(\mathbf{x}_{o, i}^{T} \mathbf{u}_{n, j}+\mathbf{x}_{n, i}^{T} \mathbf{u}_{o, j}+\mathbf{x}_{o, j}^{T} \mathbf{u}_{n, i}+\mathbf{x}_{n, j}^{T} \mathbf{u}_{o, i}\right) / 2$ is the bending strain.

The conditions of rigid director $\mathbf{x}_{n}^{T} \mathbf{u}_{n}=0$ and vanishing shear strain $\mathrm{e}_{\mathrm{zx}}^{0}=\mathrm{e}_{\mathrm{xh}}^{0}=0$ yield:

$$
\mathbf{u}_{n}=-\left[\begin{array}{c}
\mathbf{x}_{n}^{T} \\
\mathbf{x}_{o, \mathrm{x}}^{T} \\
\mathbf{x}_{o, \mathrm{~h}}^{T}
\end{array}\right]^{-1}\left\{\begin{array}{c}
0 \\
\mathbf{x}_{n}^{T} \mathbf{u}_{o, \mathrm{x}} \\
\mathbf{x}_{n}^{T} \mathbf{u}_{o, \mathrm{~h}}
\end{array}\right\}=\frac{1}{j}\left[\left(-\mathbf{x}_{o, \mathrm{~h}} \times \mathbf{x}_{n}\right)\left(\mathbf{x}_{n}^{T} \mathbf{u}_{o, \mathrm{x}}\right)+\left(\mathbf{x}_{o, \mathrm{x}} \times \mathbf{x}_{n}\right)\left(\mathbf{x}_{n}^{T} \mathbf{u}_{o, \mathrm{~h}}\right)\right]
$$

which is orthogonal to $\mathbf{x}_{n}$. By making use of $\mathrm{e}_{z i, j}^{o}=0$ and the last equation,

$$
\mathrm{e}_{i j}^{b}=-\mathbf{x}_{n}^{T} \mathbf{u}_{o, i j}-\mathbf{x}_{o, i j}^{T} \mathbf{u}_{n}=-\mathbf{x}_{n}^{T} \mathbf{u}_{o, i j}+\frac{1}{j}\left(\mathbf{x}_{o, i j} \times \mathbf{x}_{o, \mathrm{~h}}\right)^{T} \mathbf{x}_{n} \mathbf{x}_{n}{ }^{T} \mathbf{u}_{o, \mathrm{x}}-\frac{1}{j}\left(\mathbf{x}_{o, i j} \times \mathbf{x}_{o, \mathrm{x}}\right)^{T} \mathbf{x}_{n} \mathbf{x}_{n}{ }^{T} \mathbf{u}_{o, \mathrm{~h}}
$$

Let $\mathbf{e}_{1}$ and $\mathbf{e}_{2}$ be mutually perpendicular unit vectors tangential to $\mathbf{x}_{o}$, the vectors of physical membrane and bending strain components with respect to the $\boldsymbol{e}_{1}$ - and $\boldsymbol{e}_{2}$-directions can be obtained as:

$$
\left\{\begin{array}{c}
\mathrm{e}_{11}^{b} \\
\mathrm{e}_{22}^{b} \\
2 \mathrm{e}_{12}^{b}
\end{array}\right\}=\mathbf{T}\left\{\begin{array}{c}
\mathrm{e}_{\mathrm{xx}}^{b} \\
\mathrm{e}_{\mathrm{hh}}^{b} \\
2 \mathrm{e}_{\mathrm{xh}}^{b}
\end{array}\right\},\left\{\begin{array}{c}
\mathrm{e}_{11}^{m} \\
\mathrm{e}_{22}^{m} \\
2 \mathrm{e}_{12}^{m}
\end{array}\right\}=\mathbf{T}\left\{\begin{array}{c}
\mathrm{e}_{\mathrm{xx}}^{m} \\
\mathrm{e}_{\mathrm{hh}}^{m} \\
2 \mathrm{e}_{\mathrm{xh}}^{m}
\end{array}\right\}
$$

where

$$
\mathbf{T}=\left[\begin{array}{cccccccc}
e_{1}^{T} x_{0, \mathrm{x}} & e_{1}^{T} x_{0, \mathrm{x}} & e_{2}^{T} x_{0, \mathrm{x}} e_{2}^{T} x_{0, \mathrm{x}} & e_{1}^{T} x_{0, \mathrm{x}} e_{2}^{T} x_{0, \mathrm{x}} \\
e_{1}^{T} x_{0, \mathrm{~h}} e_{1}^{T} x_{0, \mathrm{~h}} & e_{2}^{T} x_{0, \mathrm{~h}} e_{2}^{T} x_{0, \mathrm{~h}} & e_{1}^{T} x_{0, \mathrm{~h}} e_{2}^{T} x_{0, \mathrm{~h}} \\
2 e_{1}^{T} x_{0, \mathrm{x}} e_{1}^{T} x_{0, \mathrm{~h}} & 2 e_{2}^{T} x_{0, \mathrm{x}} e_{2}^{T} x_{0, \mathrm{~h}} & e_{1}^{T} x_{0, \mathrm{x}} e_{2}^{T} x_{0, \mathrm{~h}}+e_{2}^{T} x_{0, \mathrm{x}} e_{1}^{T} x_{0, \mathrm{~h}}
\end{array}\right]^{-1}
$$




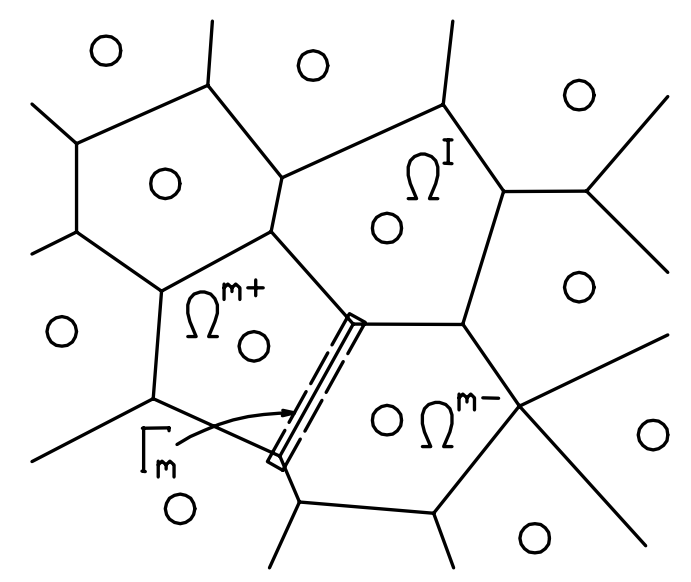

Figure 1. In nodal integration for mesh-free methods, Voronoi cells can be the nodal subdomains. The representative points in the cells are nodes $\bigcirc . \Gamma_{m}$ denotes the common boundary segment of subdomains $\Omega^{m+}$ and $\Omega^{m-}$.

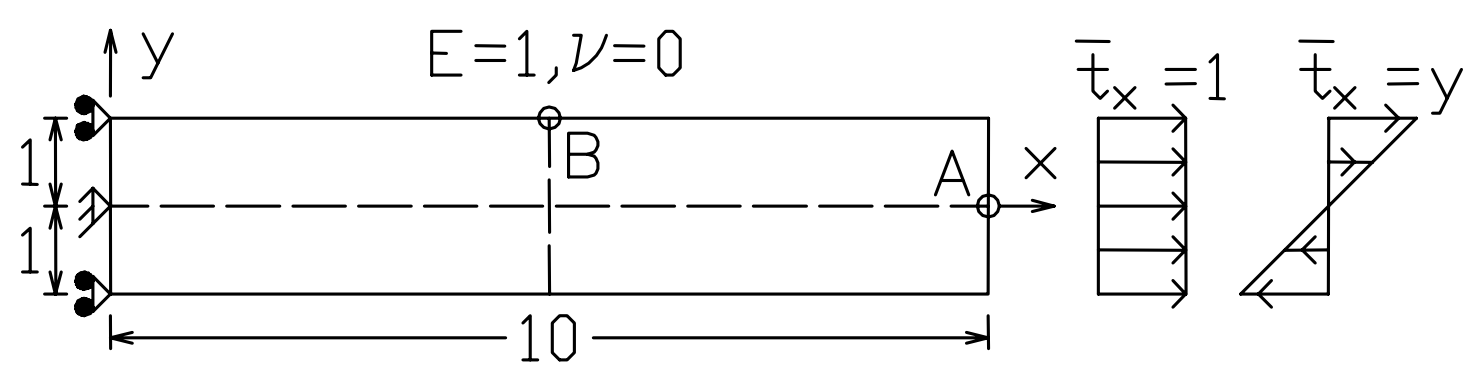

Figure 2. A $10 \times 2$ rectangular panel divided into four integration subdomains. 


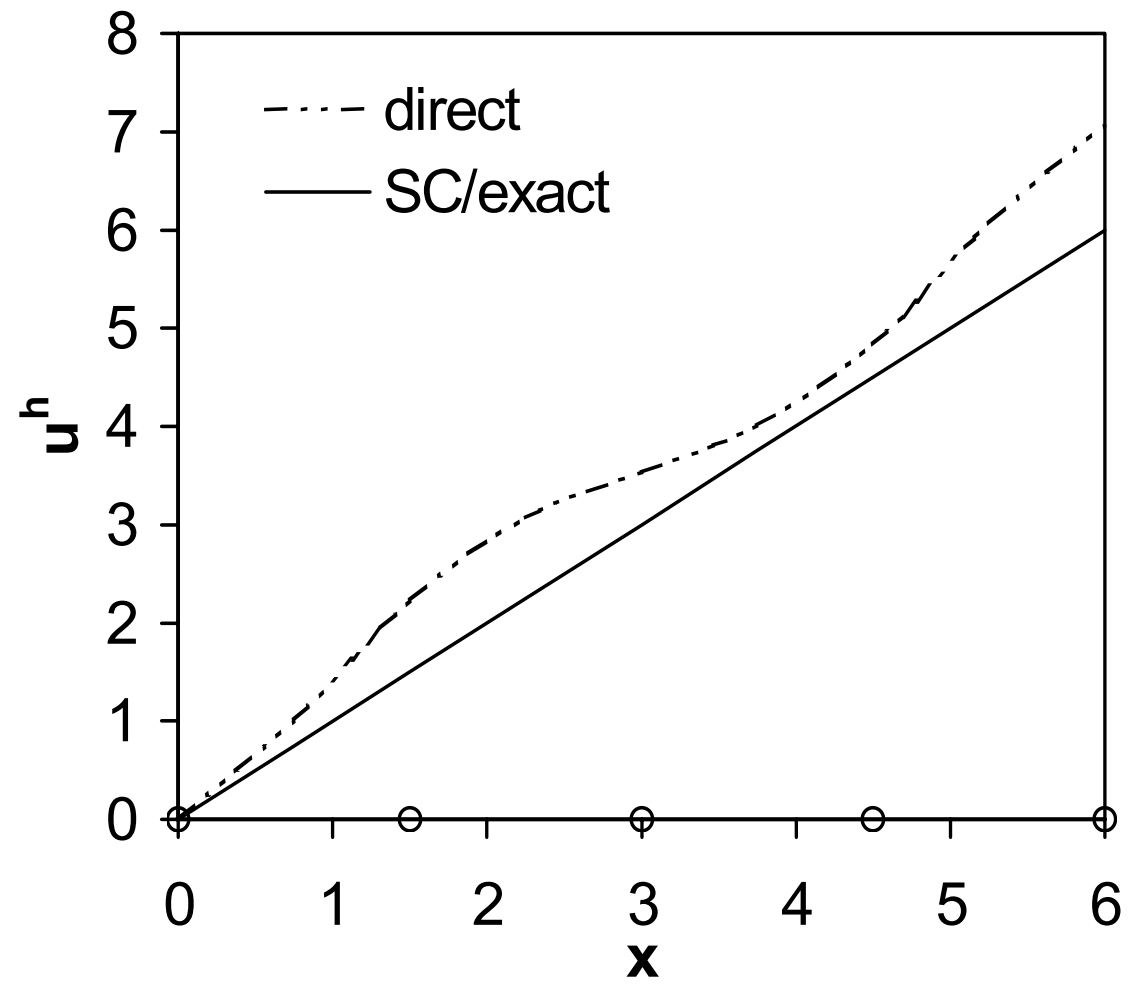

Figure 3. Predictions for the zero body force problem. The trial solution is the five-node interpolation function and $O$ 's along $x$-axis denote nodal locations.

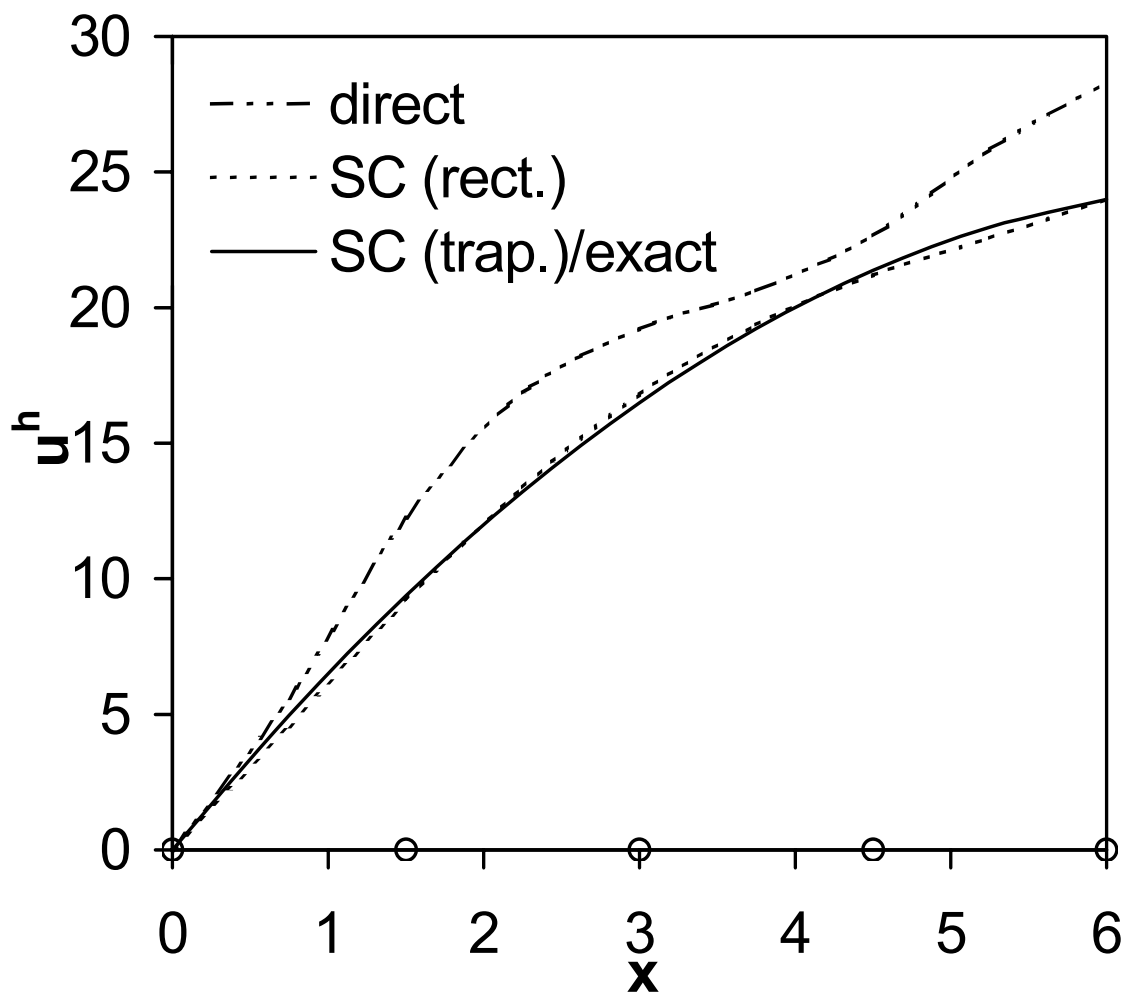

Figure 4. Predictions for the constant body force problem. The trial solution is the fivenode interpolation function and $O$ 's along $\mathrm{x}$-axis denote nodal locations. 


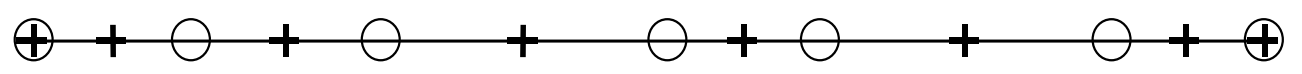

Figure 5. O's are nodes and +'s are node-to-node midpoints. O's and +'s are the sampling points of the body forces in SC nodal (rect.) and SC nodal (trap.), respectively.

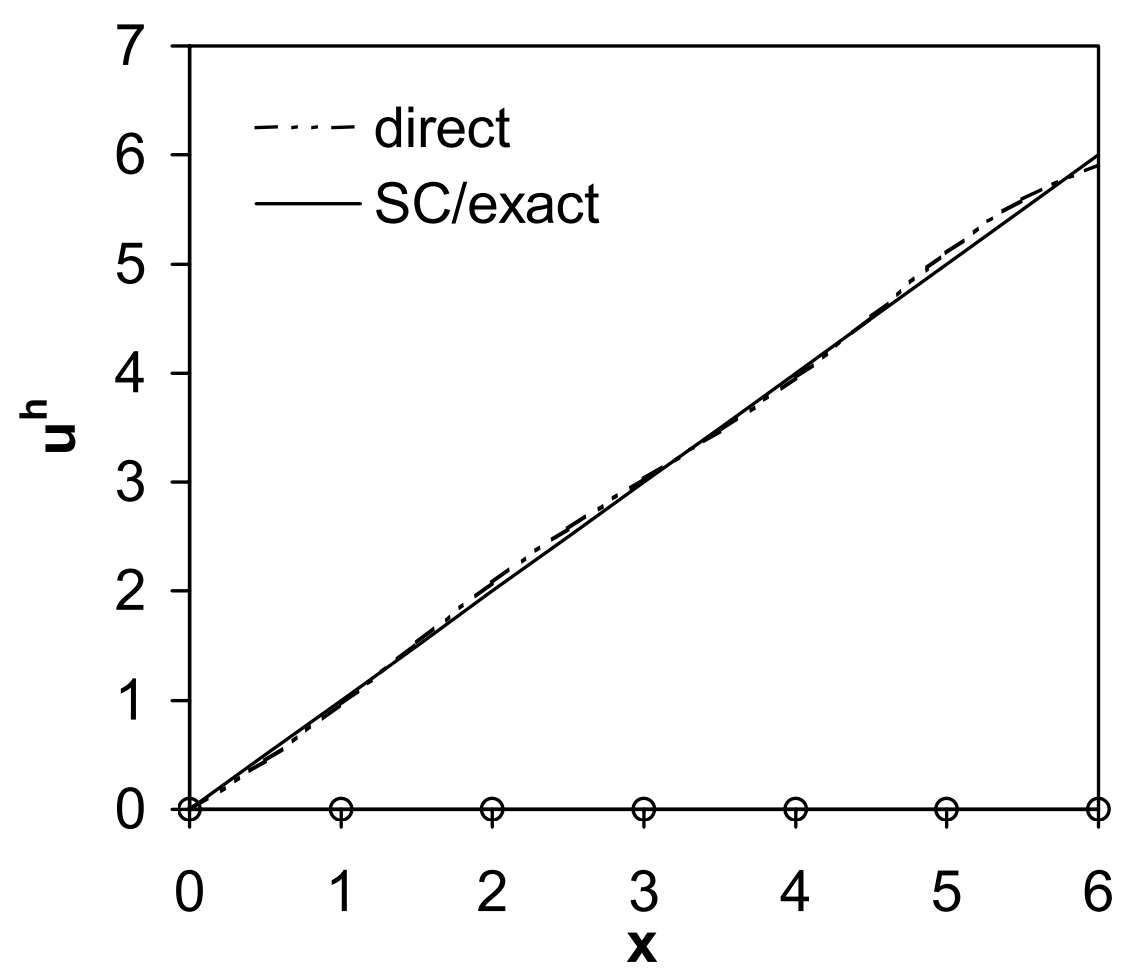

Figure 6. Predictions for the zero body force problem. The trial solution is obtained by MLS with $\mathrm{Nn}=7, \mathrm{~Np}=2$ and $\mathrm{R}=3 \mathrm{Ln}$. $\mathrm{O}$ 's along the $\mathrm{x}$-axis denote nodal locations. 


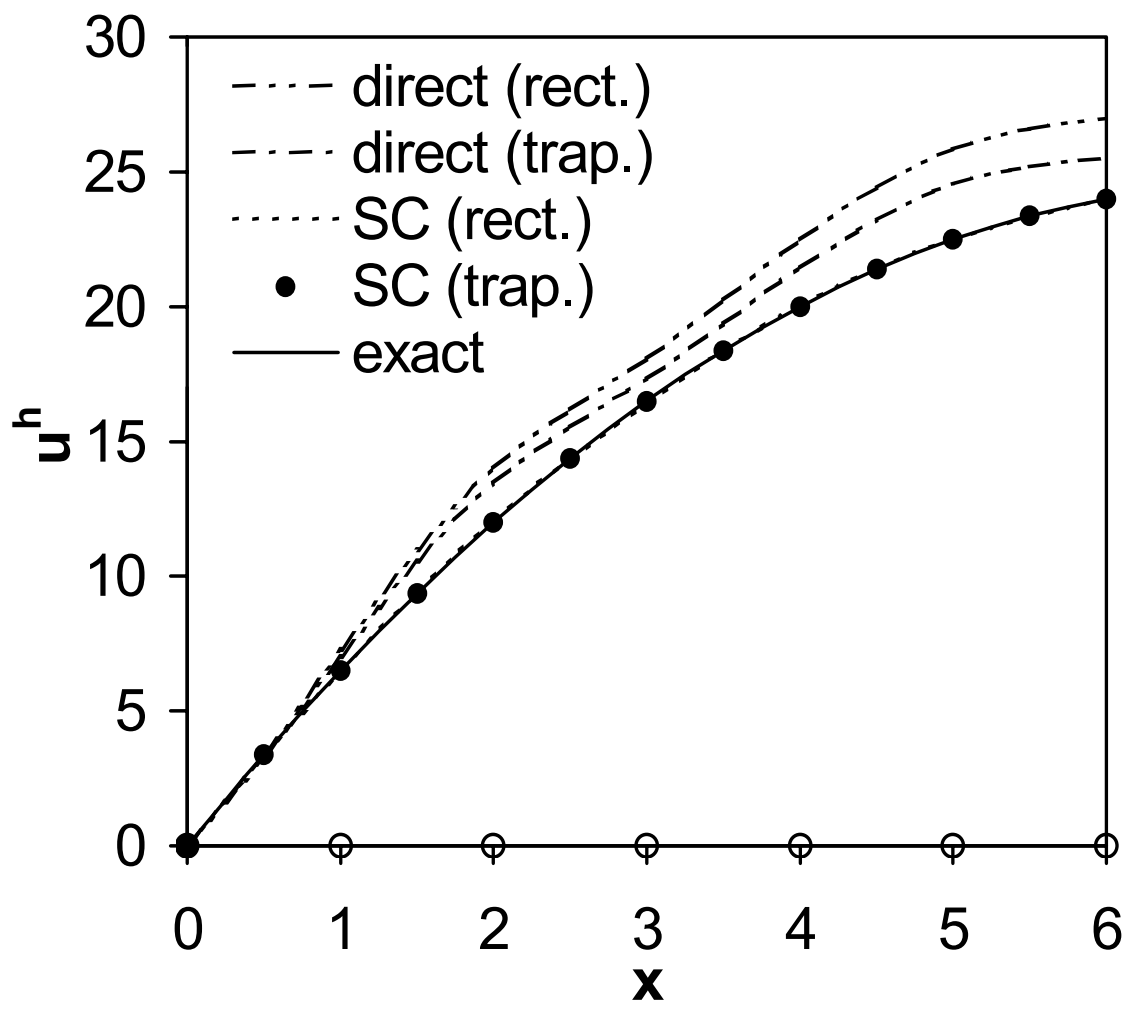

Figure 7. Predictions for the constant body force problem. The trial solution is obtained by MLS with $\mathrm{Nn}=7, \mathrm{~Np}=2$ and $\mathrm{R}=3 \mathrm{Ln}$. $\mathrm{O}$ 's along the $\mathrm{x}$-axis denote nodal locations.

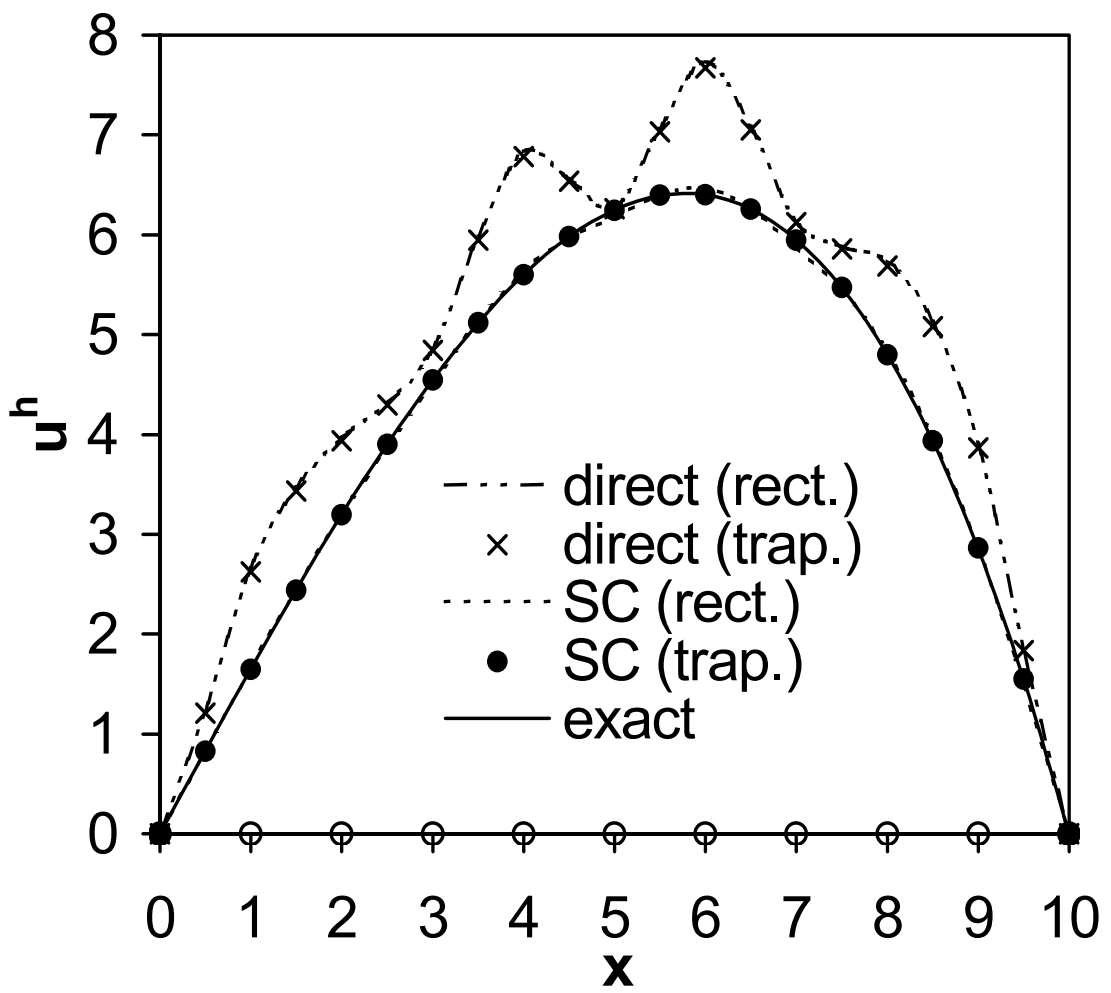

Figure 8. Effect of rectangular and trapezoidal rules in accounting for linear body force. The trial solution is obtained by MLS with $\mathrm{Nn}=11, \mathrm{~Np}=2$ and $\mathrm{R}=3 \mathrm{Ln}$. $\mathrm{O}$ 's along the $\mathrm{x}$-axis denote nodal locations. 


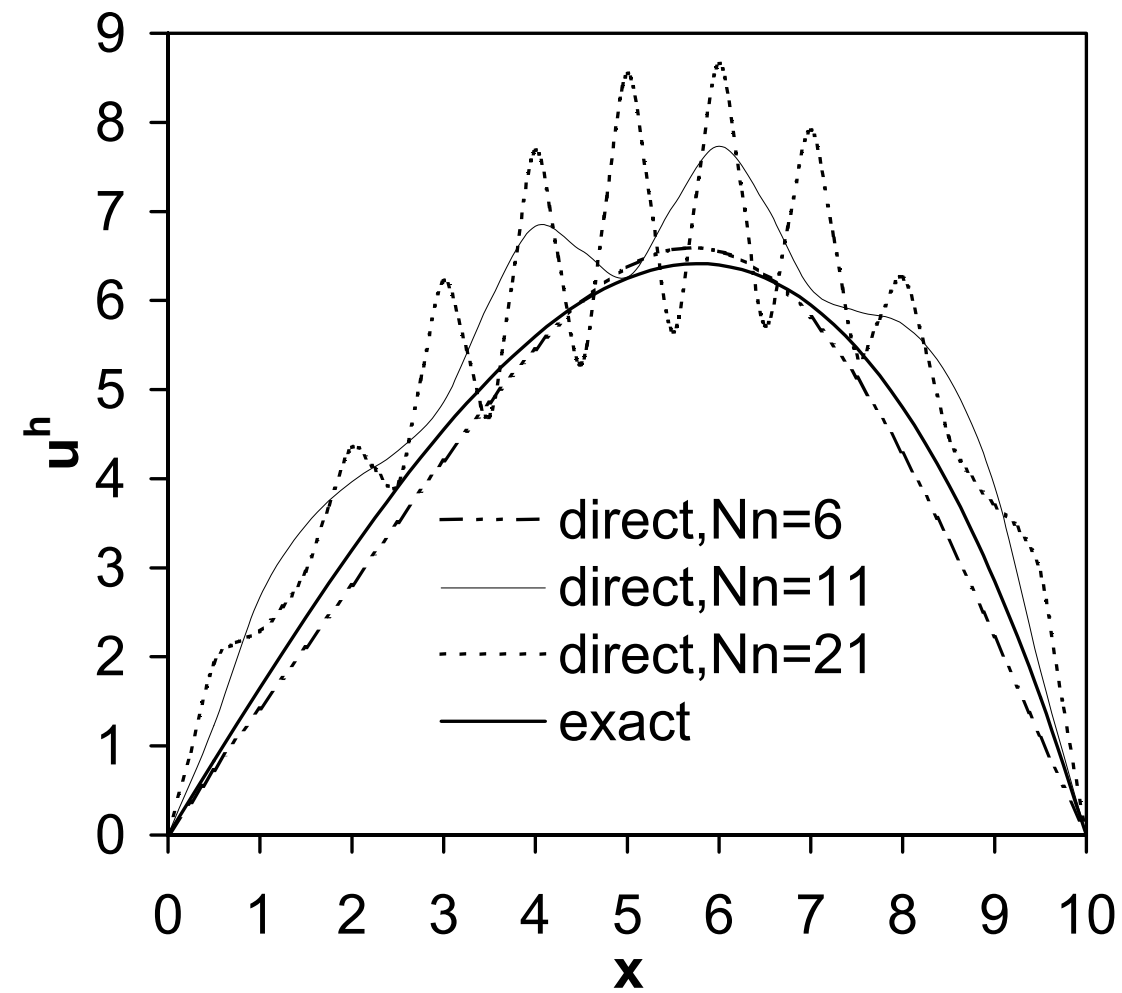

Figure 9. Predictions of direct nodal integration for the linear body force problem. The trial solutions are obtained by MLS with $\mathrm{Np}=2$ and $\mathrm{R}=3 \mathrm{Ln}$.

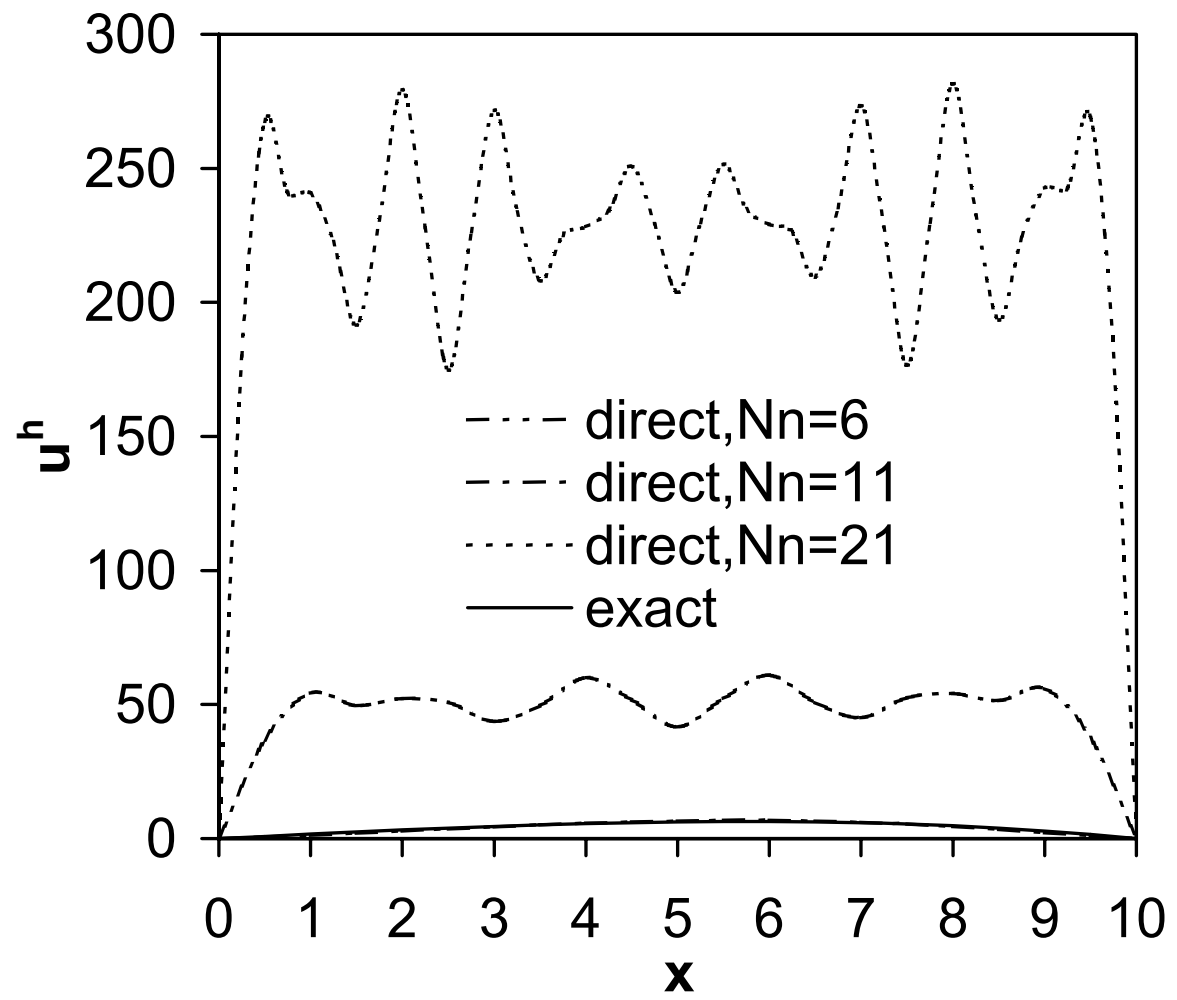

Figure 10. Predictions of direct nodal integration for the linear body force problem. The trial solutions are obtained by MLS with $\mathrm{Np}=3$ and $\mathrm{R}=4 \mathrm{Ln}$. 


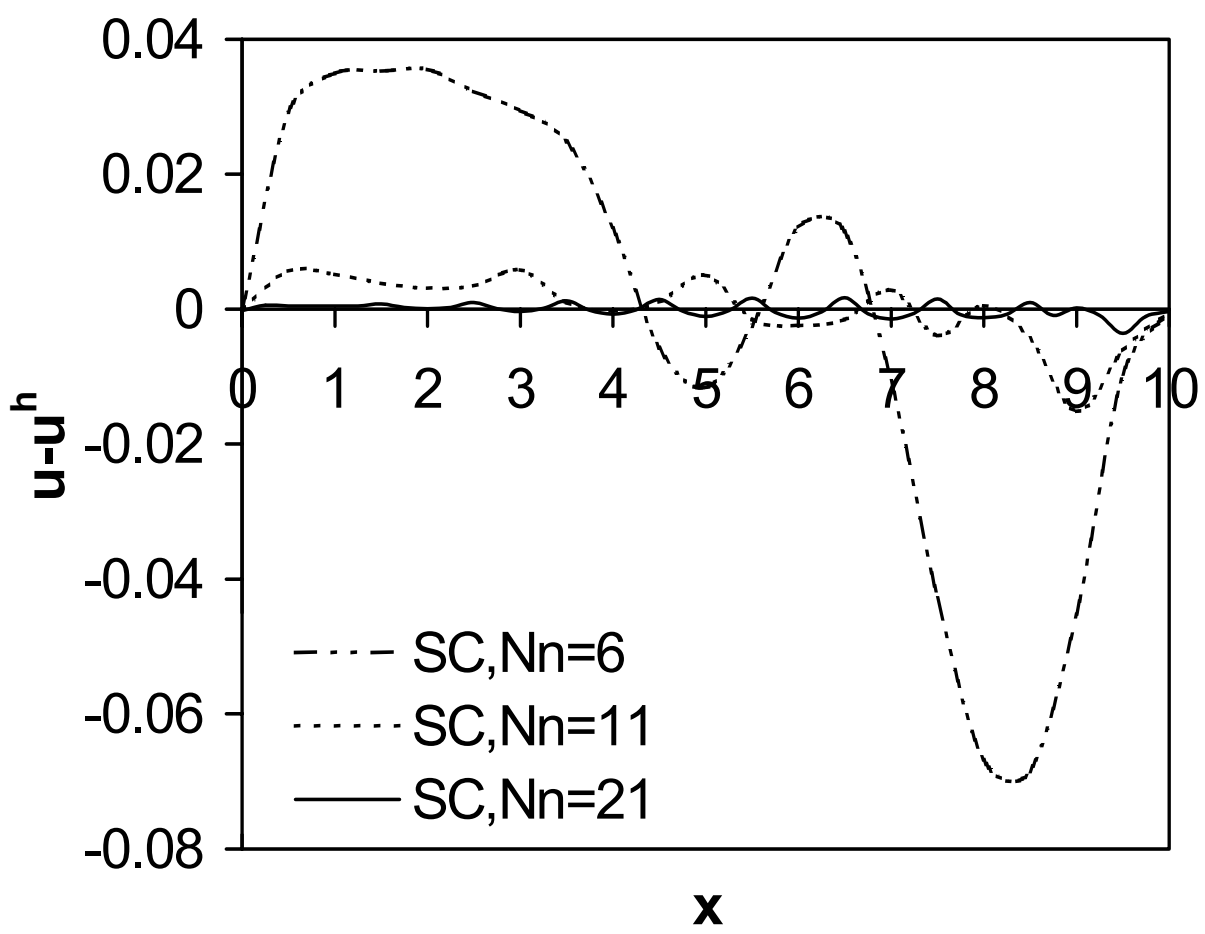

Figure 11. Errors of SC nodal integration for the linear body force problem. The trial solutions are obtained by MLS with $\mathrm{Np}=2$ and $\mathrm{R}=3 \mathrm{Ln}$.

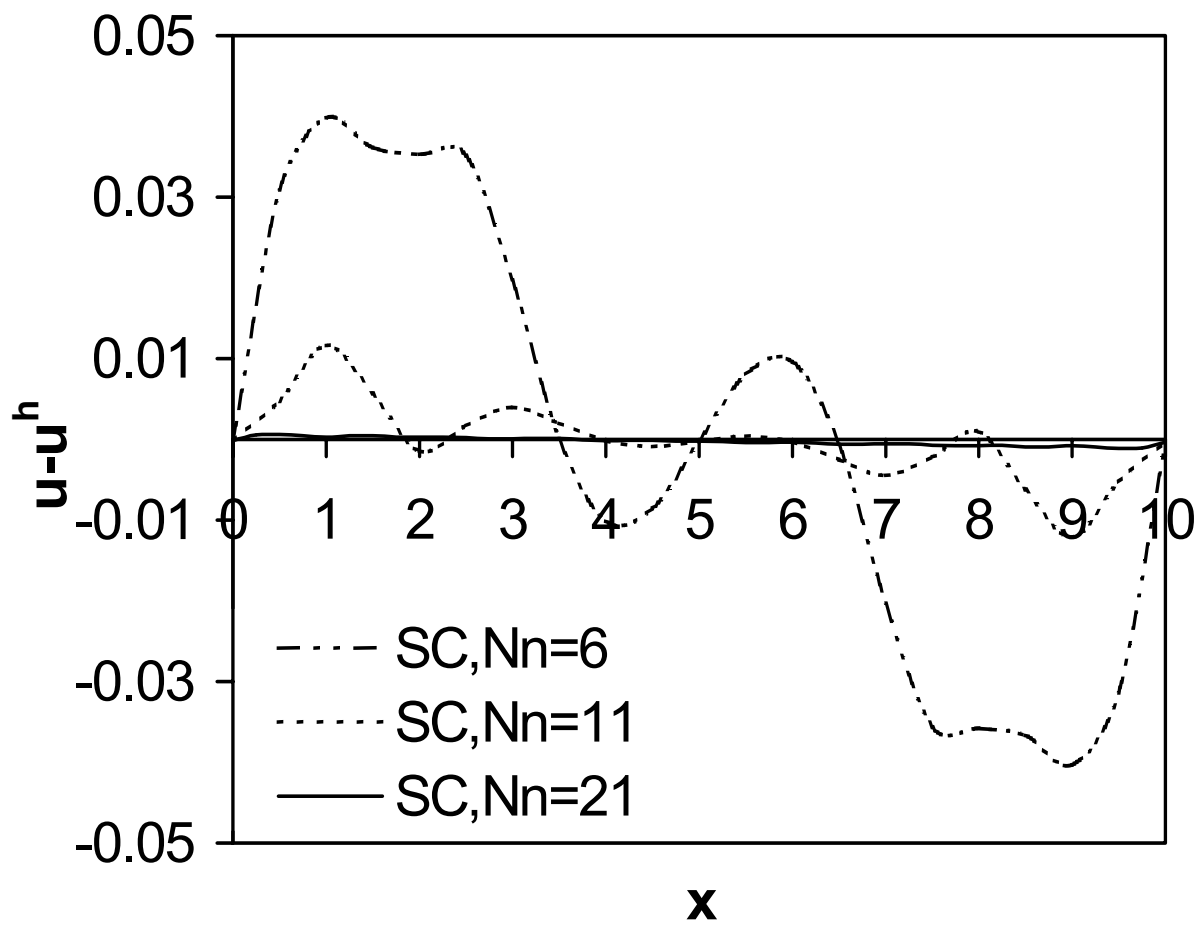

Figure 12. Errors of SC nodal integration for the linear body force problem. The trial solutions are obtained by MLS with $\mathrm{Np}=3$ and $\mathrm{R}=4 \mathrm{Ln}$. 


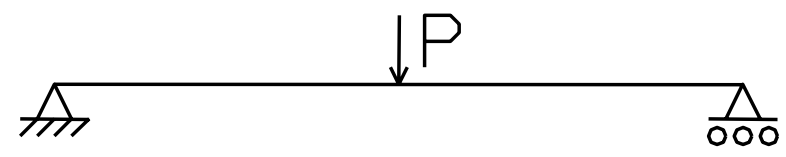

Figure 13. A simply supported beam loaded at its mid-span.

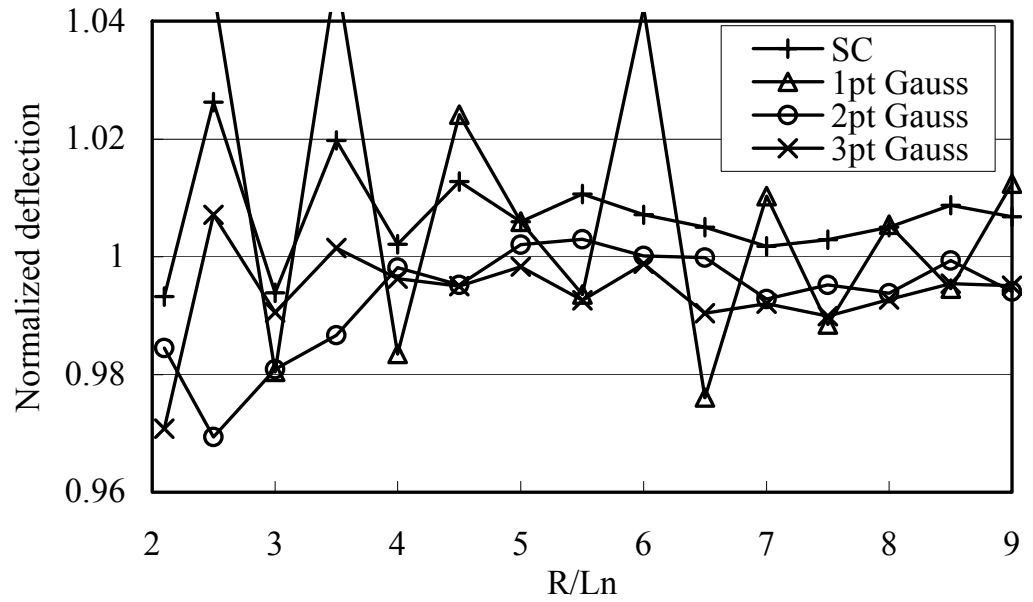

(a) $\mathrm{Np}=2$ and $\mathrm{Nn}=11$

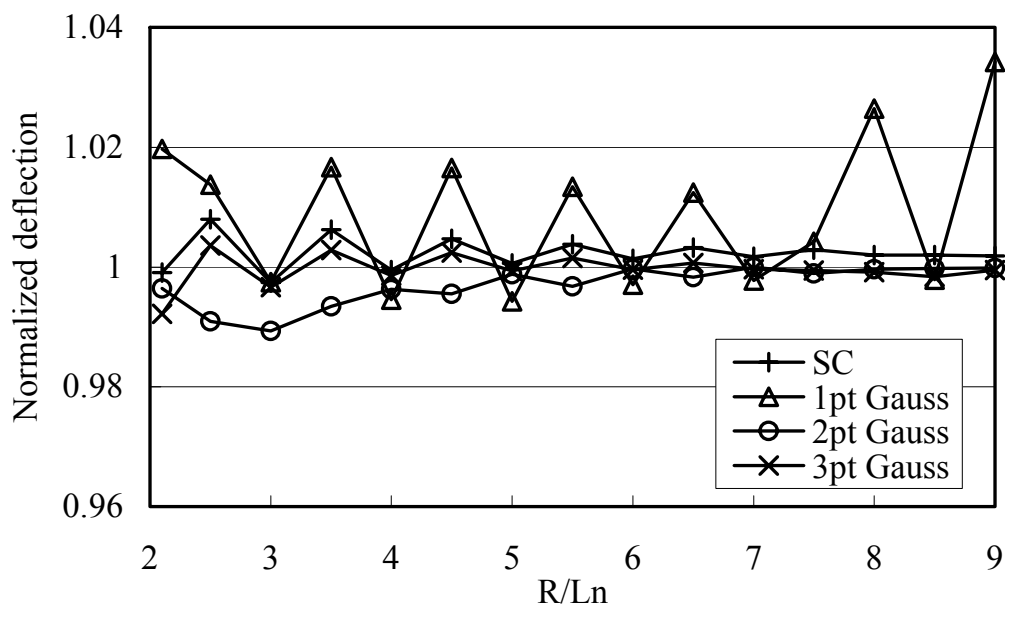

(b) $\mathrm{Np}=2$ and $\mathrm{Nn}=21$

Figure 14. Normalized deflection under $\mathrm{P}$ for (a) $\mathrm{Nn}=11$ and (b) $\mathrm{Nn}=21$, see Figure 13. 


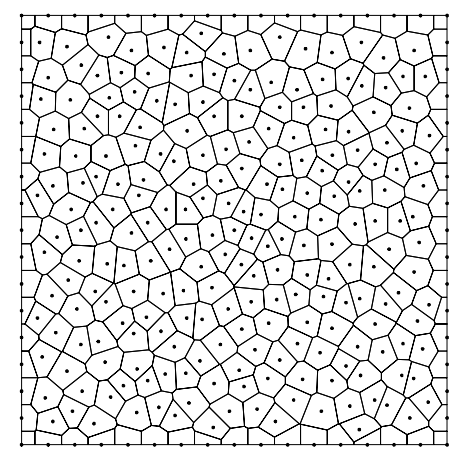

Figure 15. The Voronoi cells for a simply square plate modeled by 17 $\square 17$ nodes.

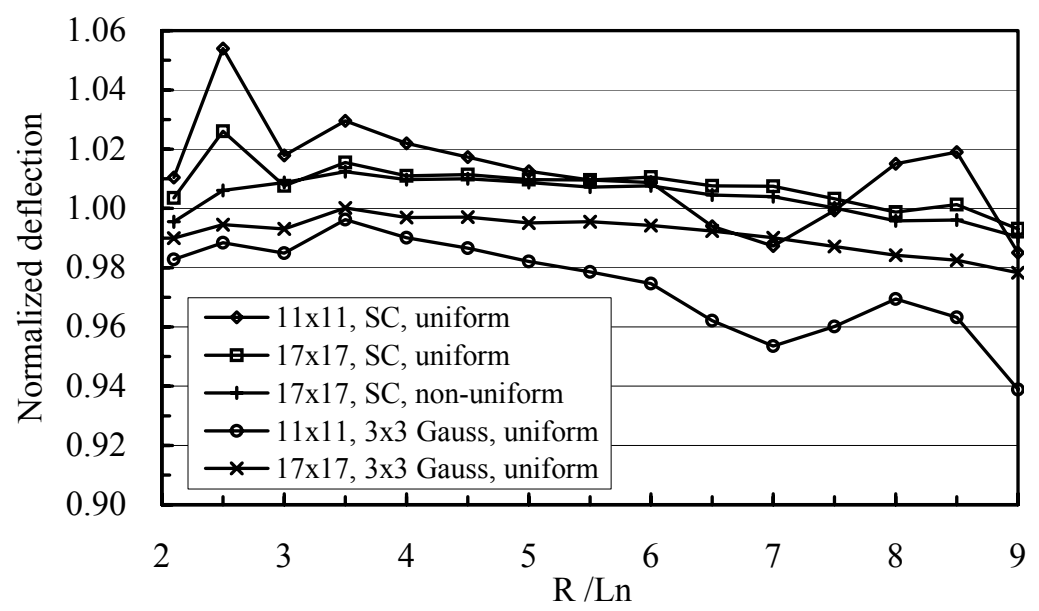

Figure 16. Normalized central deflection for a simply square plate subjected to a central point load, quadratic basis is employed.

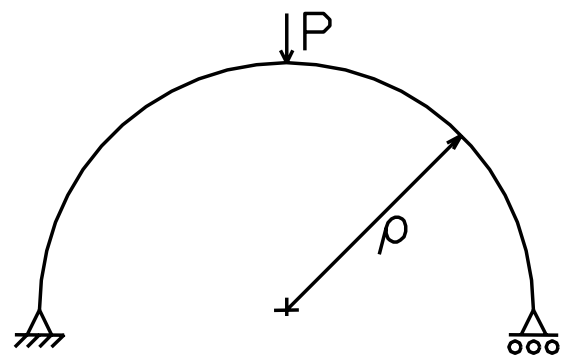

Figure 15. A simply supported arc loaded at its mid-span. 


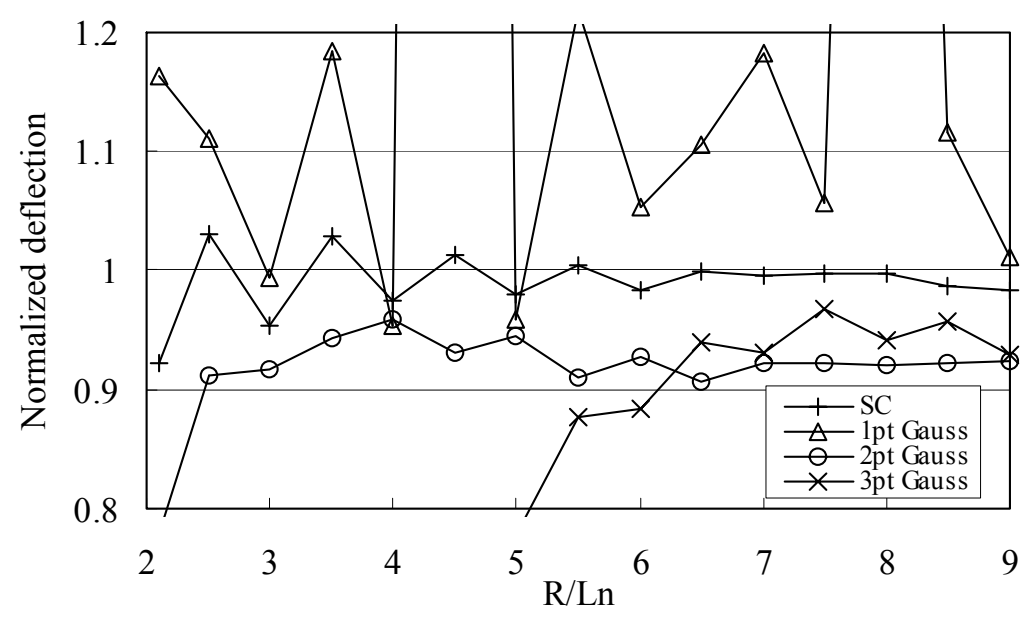

(a) $\mathrm{Np}=2, \mathrm{Nn}=15, \odot / h=50$

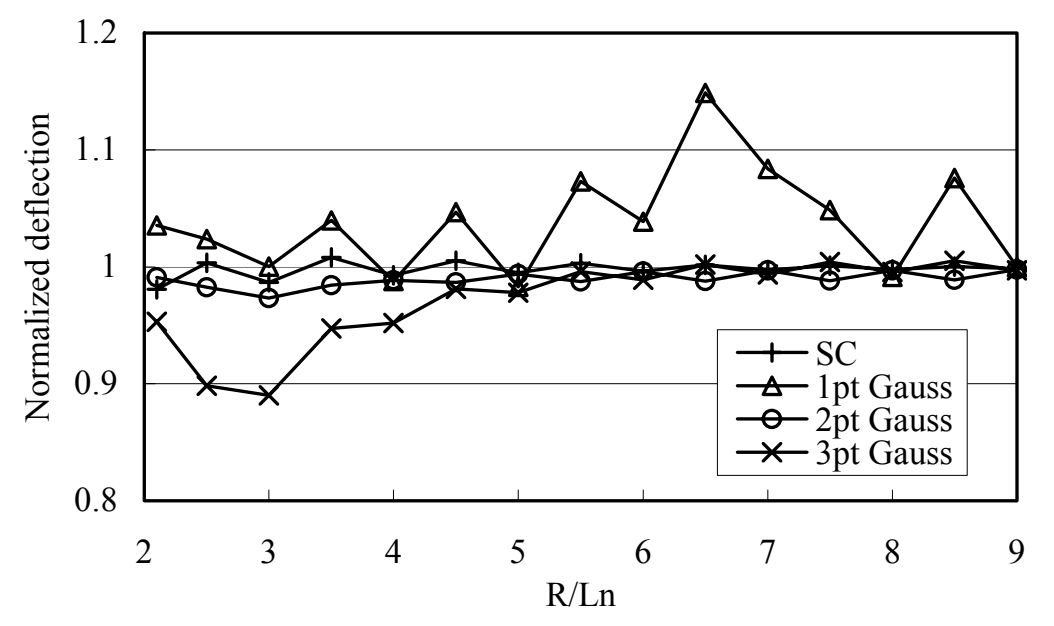

(b) $\mathrm{Np}=2, \mathrm{Nn}=31, \odot / h=50$

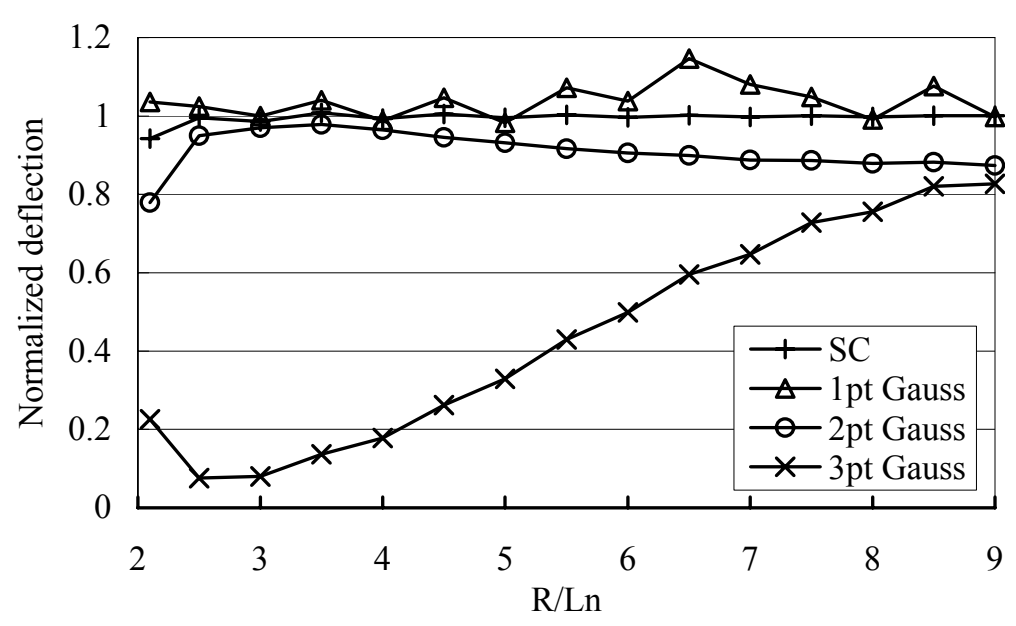

(b) $\mathrm{Np}=2, \mathrm{Nn}=31, \odot / h=500$

Figure 16. Normalized deflections under the loading force in a simply supported arc, see Figure 15. 